\title{
Interaction of wave with a body floating on a wide polynya
}

Z. F. Li, Y. Y. Shi, and G. X. Wu

Citation: Physics of Fluids 29, 097104 (2017); doi: 10.1063/1.4991675

View online: http://dx.doi.org/10.1063/1.4991675

View Table of Contents: http://aip.scitation.org/toc/phf/29/9

Published by the American Institute of Physics

\section{Articles you may be interested in}

Experimental and numerical study on bubble-sphere interaction near a rigid wall

Physics of Fluids 29, 092102 (2017); 10.1063/1.4993800

The propulsion of two flapping foils with tandem configuration and vortex interactions

Physics of Fluids 29, 097102 (2017); 10.1063/1.5001501

The regime of large bubble entrapment during a single drop impact on a liquid pool

Physics of Fluids 29, 092101 (2017); 10.1063/1.4992124

Heat transport and coupling modes in Rayleigh-Bénard convection occurring between two layers with largely different viscosities

Physics of Fluids 29, 096602 (2017); 10.1063/1.4989592

Instability of a binary liquid film flowing down a slippery heated plate

Physics of Fluids 29, 092105 (2017); 10.1063/1.4989558

Novel criteria for the development of monotonic and oscillatory instabilities in a two-layer film

Physics of Fluids 29, 092104 (2017); 10.1063/1.5001729

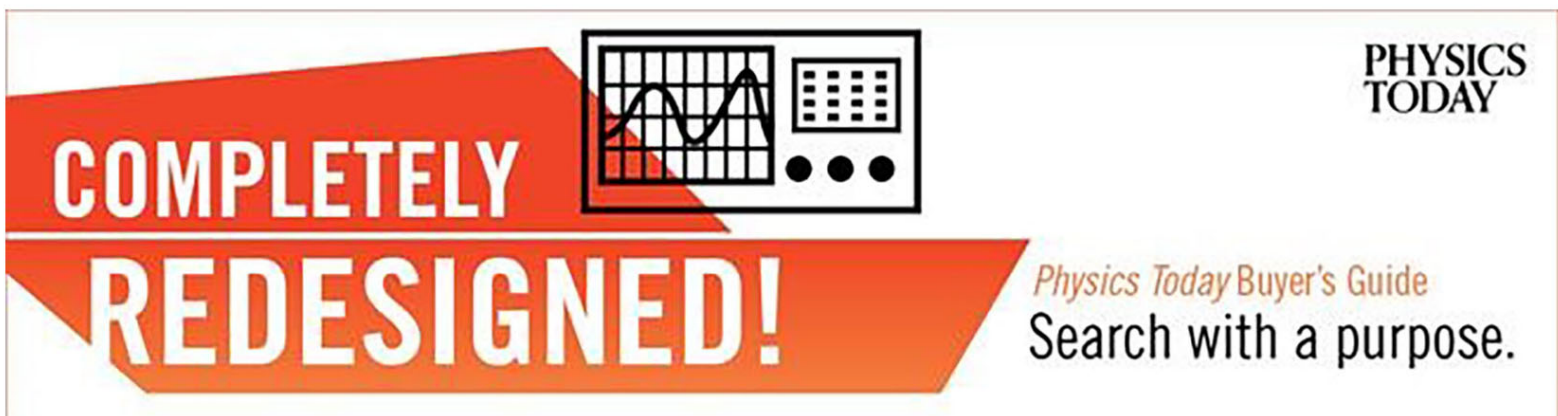




\title{
Interaction of wave with a body floating on a wide polynya
}

\author{
Z. F. Li, ${ }^{1}$ Y. Y. Shi, ${ }^{2}$ and G. X. Wu ${ }^{1,3, a)}$ \\ ${ }^{1}$ School of Naval Architecture and Ocean Engineering, Jiangsu University of Science and Technology, \\ Zhenjiang 212003, China \\ ${ }^{2}$ College of Shipbuilding Engineering, Harbin Engineering University, Harbin 150001, China \\ ${ }^{3}$ Department of Mechanical Engineering, University College London, Torrington Place, London WC1E 7JE, \\ United Kingdom
}

(Received 22 June 2017; accepted 28 August 2017; published online 20 September 2017)

\begin{abstract}
A method based on wide spacing approximation is proposed for the interaction of water wave with a body floating on a polynya. The ice sheet is modelled as an elastic plate and fluid flow is described by the velocity potential theory. The solution procedure is constructed based on the assumption that when the distance between two disturbances to the free surface is sufficiently large, the interactions between them involve only the travelling waves caused by the disturbances and the effect of the evanescent waves is ignored. The solution for the problem can then be obtained from those for a floating body without an ice sheet and for an ice sheet/free surface without a floating body. Both latter solutions have already been found previously and therefore there will be no additional effort in solution once the wide spacing approximation formulation is derived. Extensive numerical results are provided to show that the method is very accurate compared with the exact solution. The obtained formulations are then used to provide some insightful explanations for the physics of flow behaviour, as well as the mechanism for the highly oscillatory features of the hydrodynamic force and body motion. Some explicit equations are derived to show zero reflection by the polynya and peaks and troughs of the force and excited body motion. It is revealed that some of the peaks of the body motion are due to resonance while others are due to the wave characters in the polynya. Published by AIP Publishing. https://doi.org/10.1063/1.4991675
\end{abstract}

\section{INTRODUCTION}

Interaction of water wave with a floating body has been of great interest due to the complexity of flow features and its practical relevance, in particular to ocean and coastal engineering, as well as naval architecture. The ocean surface is usually treated as infinitely large, on which the pressure is assumed to be atmospheric, and it is commonly refereed as the free surface when the atmospheric pressure is taken as constant. The research over the last decades has significantly advanced our understanding of the nature of the wave physics and the mechanism of its interaction with a floating body. The latest development in Arctic engineering, in particular the possibility of new shipping routes in the next few decades, has led to some new technical challenges. One of such challenges arises when a ship navigates through a strip of water confined between large ice sheets, which could be formed through melting of Arctic ice ${ }^{1}$ or opened up by an icebreaker. ${ }^{2}$ The flow and body motion features will be different from those in open sea and will very much depend on the wave/ice/body interaction. A better understanding of these features is highly important for safety, environmental protection as well as economic cost.

The observations by Robin ${ }^{3}$ suggested that a large ice sheet could be treated as an elastic plate in the wave/ice interaction problems. This model has been widely used subsequently. A review of earlier work for this kind of problem was given

a)Author to whom correspondence should be addressed: g.wu@ucl.ac.uk. Tel.: +4420 7679 3870. Fax: +44 2073880180 . by Squire et al., ${ }^{4}$ and the more recent ones were given by Squire. ${ }^{1,5}$ A semi-infinite ice sheet on the free surface was considered based on the thin plate model by Fox and Squire ${ }^{6}$ and based on the thick plate model by Fox and Squire ${ }^{7}$ using the matched eigenfunction expansion method. Numerical comparison showed that in terms of the reflection and transmission coefficients these two models gave graphically indistinguishable results. The case was then extended to the oblique incident wave by Fox and Squire. ${ }^{8}$ For the similar problems, Sahoo et $a .^{9}{ }^{9}$ introduced an inner product of orthogonality and considered the ice sheets with various edge conditions. Meylan and Squire ${ }^{10}$ adopted the Green function method which was more flexible and could be applied to a much wider range of problems. It is also possible to apply the Wiener-Hopf method for this type of problem. ${ }^{11}$ Chung and Fox ${ }^{12}$ used the method for the oblique reflection and transmission of ocean waves into the semi-infinite ice sheet. Other notable work using the WienerHopf method includes those by Balmforth and Craster ${ }^{13}$ and by Tkacheva. ${ }^{14,15}$ Chung and Linton ${ }^{16}$ considered wave reflection and transmission when propagating across a gap between two semi-infinite ice sheets, or polynya, and found that the reflection coefficient could be zero at discrete frequencies. Williams and Squire ${ }^{17}$ solved the problem of interaction of wave with three connected plates of different thickness. When the thickness is taken as zero, it becomes a free surface and thus the polynya can be treated as one of the special cases of such a problem. The problem of an imperfect ice sheet, with a crack, for example, was investigated by Evans and Porter, ${ }^{18}$ Porter and Evans, ${ }^{19}$ and more recently by Sturova and Tkacheva. ${ }^{20}$ 
The above work is mainly about the interactions between ocean waves and ice sheets. For wave/ice/body interaction problems, Das and Mandal ${ }^{21}$ studied the oblique wave scattering by a circular cylinder submerged beneath an ice cover through the multipole expansion method. Sturova ${ }^{22}$ considered the problem of a submerged cylinder and the corresponding Green function satisfying all the boundary conditions apart from that on the body surface was derived. The method was then extended to the problem of two semi-infinite ice sheets connected by vertical and flexural rotational springs ${ }^{23}$ and the ice floe or polynya. ${ }^{24}$ For a floating body on the polynya, Ren et $a l^{25}$ obtained the semi-analytical solution based on the matched eigenfunction expansions for a rectangular box. Li et al. ${ }^{26}$ considered the nonlinear effects of the body motion through a semi-analytical solution for a circular cylinder in large amplitude oscillation. For general cases of a body with arbitrary shapes, Li et al. ${ }^{27}$ developed a hybrid method by combining the boundary element method and eigenfunction expansion method.

The problem described above have been mainly solved exactly in the sense when a discretization of the boundary is refined or the number of terms in an infinite series further increases, the numerical result no longer changes within the desired accuracy. We may also notice that the solution procedure for such a problem is much more complex than that for a free surface without an ice sheet or for an ice sheet without a free surface. This is reflected by the far more complex Green function for the wave/body/ice interaction problem. ${ }^{24}$ Thus this has motivated the present work to develop an efficient yet highly accurate method.

Here we notice the fact that the wave generated/disturbed by the body or ice edge has two components. One is the evanescent wave which will decay exponentially away from the disturbance, while the other is the travelling wave which will propagate away from the disturbance to infinity. Thus when the locations of two disturbances are sufficiently large, only the latter needs to be considered in their interactions. Therefore, in this work by following the wide spacing approximation used in the multi-bodies/wave interaction, ${ }^{28}$ we consider the problem of wave interaction with a body floating on a wide polynya. The wide approximation enables us to construct a solution based on those for the problem of a floating body without an ice sheet and the problem of an ice sheet/free surface without a floating body. The merit of this method is that it can give an accurate solution based on what has already been solved previously. In Secs. II-IV, we shall first derive the formulation based on this method. Extensive numerical results are then provided, including the wave propagation across the polynya, and interaction with a submerged body and a floating body in polynya. The method is verified through the excellent agreement with the exact solution. The formulation is subsequently used to provide deep insights into the complex wave features, as well as hydrodynamic forces and body response to the waves.

\section{MATHEMATICAL MODEL AND NUMERICAL PROCEDURES}

\section{A. Mathematical model}

We consider the interaction problem of wave with a twodimensional body floating on a wide polynya confined between two semi-infinite ice sheets, as sketched in Fig. 1. A Cartesian coordinate system $\vec{x}=(x, z)$ fixed in space is chosen with the origin $O$ at the undisturbed mean free surface, $x$ being the horizontal direction and $z$ vertically upward. When the body is at its equilibrium position, the $z$-axis passes through the centre of its mass. In each side of the polynya, i.e., $x<x_{1}$ and $x>x_{2}$, the upper surface of the fluid is covered by a semi-infinite ice sheet. The width of the polynya is $l=x_{2}-x_{1}$. The body with beam $a$ and draught $b$, respectively, is assumed to be excited into motion by an incident wave propagating underneath the left ice sheet. The present work is undertaken on the basis that the gap between the edge of the ice to the body is much larger than the typical dimension of the body or $l \gg a$.

The fluid with density $\rho$ and constant depth $H$ is assumed to be inviscid, incompressible, and homogeneous, and its motion to be irrotational. Thus the velocity potential $\Phi$ can be introduced to describe the fluid flow. Under the assumption that the amplitude of wave motion is small compared to its length and the dimension of the body, the linearized velocity potential theory can be further used. When the motion is sinusoidal in time with radian frequency $\omega$, the total potential can be written as ${ }^{29}$

$$
\begin{aligned}
\Phi(x, z, t)= & \operatorname{Re}\left[\alpha_{0} \phi_{0}(x, z) \mathrm{e}^{\mathrm{i} \omega t}\right] \\
& +\operatorname{Re}\left[\sum_{k=1}^{3} \mathrm{i} \omega \alpha_{k} \phi_{k}(x, z) \mathrm{e}^{\mathrm{i} \omega t}\right],
\end{aligned}
$$



FIG. 1. Coordinate system and sketch of the problem. 
where $\phi_{0}$ contains the incident potential $\phi_{I}$ and diffracted potential $\phi_{D}, \alpha_{0}$ is the amplitude of the incident wave, $\phi_{k}$ $(k=1,2,3)$ are the radiation potentials due to body oscillation with complex amplitude $\alpha_{k}$ in three degrees of freedom: translations in $x$ and $z$ directions and rotation about the $y$-axis parallel to the ice sheet edge. Mass conservation requires that the potential $\phi_{k}$ satisfies the Laplace's equation,

$$
\nabla^{2} \phi_{k}=0,(k=0,1,2,3),
$$

throughout the fluid. The combination of the linearized dynamic and kinematic free surface boundary conditions gives

$$
-\omega^{2} \phi_{k}+g \frac{\partial \phi_{k}}{\partial z}=0,\left(x_{1}<x<x_{2}, \quad z=0\right)
$$

where $g$ is the acceleration due to gravity. The ice sheet is modelled as a continuous elastic plate with uniform properties, i.e., thickness $h_{j}$, draught $d_{j}$, density $\rho_{j}$, Young's modulus $E_{j}$, and Poisson's ratio $v_{j}$ are all constants. Thus the boundary condition on the ice sheets can be written as ${ }^{6}$

$$
\begin{aligned}
& \left(L_{j} \frac{\partial^{4}}{\partial x^{4}}-m_{j} \omega^{2}+\rho g\right) \frac{\partial \phi_{k}}{\partial z}-\rho \omega^{2} \phi_{k}=0, \\
& \left(|x| \geq\left|x_{j}\right|, z=-d_{j}, j=1,2\right),
\end{aligned}
$$

where $L_{j}=E h_{j}^{3} /\left[12\left(1-v_{j}^{2}\right)\right]$ is the effective flexural rigidity of the ice sheet and $m_{j}=h_{j} \rho_{j}$ is its mass per unit area. Without loss of generality, the end of the ice sheet is assumed to be free here. Thus the vanishing of the bending moment and shear force leads to the following two conditions on the ice sheet edge corner:

$$
\frac{\partial^{2}}{\partial x^{2}}\left(\frac{\partial \phi_{k}}{\partial z}\right)=0 \text { and } \frac{\partial^{3}}{\partial x^{3}}\left(\frac{\partial \phi_{k}}{\partial z}\right)=0, \quad\left(x=x_{j}, z=-d_{j}\right) .
$$

On the vertical surface of the ice sheet, the impermeable condition yields

$$
\frac{\partial \phi_{k}}{\partial x}=0,\left(x=x_{j}, \quad-d_{j} \leq z \leq 0\right) .
$$

The impermeable condition on the body surface is

$$
\frac{\partial \phi_{0}}{\partial n}=0 \text { and } \frac{\partial \phi_{k}}{\partial n}=n_{k},(k=1,2,3),
$$

where $n_{1}$ and $n_{2}$ are the $x, z$ components of the unit normal vector $\vec{n}$ pointing into the body, $n_{3}=\left(z-z^{\prime}\right) n_{1}-\left(x-x^{\prime}\right) n_{2}$ is the component related to the rotational mode, with $\left(x^{\prime}, z^{\prime}\right)$ as the rotational centre. The boundary condition on the flat seabed can be written as

$$
\frac{\partial \phi_{k}}{\partial z}=0,(-\infty<x<+\infty, \quad z=-H) .
$$

The radiation condition requires the wave to propagate outwards,

$$
\begin{aligned}
& \lim _{x \rightarrow+\infty}\left(\frac{\partial \phi_{k}}{\partial x}+\kappa_{0}^{(2)} \phi_{k}\right)=0, \\
& \lim _{x \rightarrow-\infty}\left(\frac{\partial \phi_{k}}{\partial x}-\kappa_{0}^{(1)} \phi_{k}\right)=0,(k=1,2,3),
\end{aligned}
$$

$\lim _{x \rightarrow+\infty}\left(\frac{\partial \phi_{D}}{\partial x}+\kappa_{0}^{(2)} \phi_{D}\right)=0, \quad \lim _{x \rightarrow-\infty}\left(\frac{\partial \phi_{D}}{\partial x}-\kappa_{0}^{(1)} \phi_{D}\right)=0$, where $\kappa_{0}^{(1)}$ and $\kappa_{0}^{(2)}$ are the purely positive imaginary roots of the dispersion equations for the ice covered regions or

$-\kappa_{0}^{(j)} \tan \left[\kappa_{0}^{(j)}\left(H-d_{j}\right)\right]=\frac{\rho \omega^{2}}{L_{j}\left(\kappa_{0}^{(j)}\right)^{4}+\rho g-m_{j} \omega^{2}},(j=1,2)$.

\section{B. Hydrodynamic force and body motion}

When the velocity potential $\phi_{k}$ is solved, the pressure can be obtained through the linearized Bernoulli equation, and the hydrodynamic force exerting on the body can be obtained directly by integrating the dynamic pressure over the mean wetted body surface. Based on the decomposition of the velocity potentials Eq. (1), the hydrodynamic force can be equivalently expressed as the wave exciting force due to unit wave amplitude,

$$
f_{E, k}=-\mathrm{i} \omega \rho \int_{S_{0}} \phi_{0}(x, z) n_{k} d S
$$

and the hydrodynamic coefficients

$$
\mu_{k j}-\mathrm{i} \frac{\lambda_{k j}}{\omega}=\rho \int_{S_{0}} \phi_{j} n_{k} d S,
$$

where $\mu_{k j}$ and $\lambda_{k j}$ are the added mass and damping coefficient, respectively.

Based on Newton's law, and taking into account the hydrostatic force due to the variation of the buoyance during body oscillation, the complex motion amplitudes $\alpha_{j}(j=1,2,3)$ can be computed through the following linear equations:

$\sum_{j=1}^{3}\left[-\omega^{2}\left(m_{k j}+\mu_{k j}\right)+\mathrm{i} \omega \lambda_{k j}+C_{k j}\right] \alpha_{j}=\alpha_{0} f_{E, k}, \quad k=1,2,3$,

where $j=1,2,3$ represent the modes sway, heave, and roll; $m_{k j}$ and $C_{k j}$ are, respectively, the body mass and hydrostatic restoring coefficients.

\section{Solution procedure}

The problem described in Eqs. (1)-(11) can be solved accurately through numerical methods generally. Here we shall use wide spacing approximation. To construct the expression for the solution, we denote the radiation and scattering velocity potentials of the body in the absence of ice sheets as $\psi_{k}^{r}$ and $\psi_{0}^{s \pm}$, respectively, where + and - correspond to that the incident wave opposite to and along the $x$-axis, respectively. We further consider the problem due to a semi-infinite ice sheet and semi-infinite free surface and define the velocity potentials as $\psi_{I c e, L}^{w 2 i}$ and $\psi_{I c e, R}^{w 2 i}$, where the superscript $w 2 i$ means that the incident wave is propagating from the open water to the ice covered region, and the subscripts $L$ and $R$ mean that the semi-infinite ice sheet is covered on the left- and righthand sides of the upper surface, respectively, i.e., $x \in(-\infty, 0]$ and $x \in[0,+\infty)$. Corresponding to these two potentials, we also define $\psi_{I c e, L}^{i 2 w}$ and $\psi_{I c e, R}^{i 2 w}$, where $i 2 w$ means that the incident wave is propagating from the ice covered region to the open water. 
The velocity potentials $\psi_{k}^{r}$ and $\psi_{0}^{s \pm}$ satisfy the following boundary condition on the body surface:

$$
\frac{\partial \psi_{k}^{r}}{\partial n}=n_{k} \text { and } \frac{\partial \psi_{0}^{s \pm}}{\partial n}=0,(k=1,2,3),
$$

and the boundary conditions in Eqs. (3) and (8), respectively, on the free surface and flat seabed. At infinity we have

$$
\begin{aligned}
& \lim _{x \rightarrow \pm \infty}\left(\frac{\partial \psi_{k}^{r}}{\partial x} \pm k_{0} \psi_{k}^{r}\right)=0,(k=1,2,3), \\
& \lim _{x \rightarrow \pm \infty}\left[\frac{\partial\left(\psi_{0}^{s \pm}-\psi_{I}\right)}{\partial x} \pm k_{0}\left(\psi_{0}^{s \pm}-\psi_{I}\right)\right]=0,
\end{aligned}
$$

where $k_{0}$ is the purely positive imaginary root of the dispersion equation for open water or

$$
-k_{0} \tan \left(k_{0} H\right)=\frac{\omega^{2}}{g}
$$

and $\psi_{I}$ is the incident potential in open water.

For the interaction problem of wave with the semi-infinite ice sheet, the velocity potential should satisfy the boundary conditions in Eqs. (3) and (4), respectively, on the free surface and the ice sheet, and the boundary condition in Eq. (8) on the flat seabed. Also, the free edge condition in Eq. (5) should be satisfied. At infinity, the radiation conditions are the same as those in Eqs. (17) and (10), in the open water and ice covered region, respectively.

Here we notice that the velocity potentials $\psi_{k}^{r}$ and $\psi_{0}^{s \pm}$ are classic problems and have been solved previously, for example, by the hybrid integral equation and eigenfunction expansion method $^{30}$ or the hybrid finite element and integral equation method. ${ }^{31}$ Similarly the velocity potential $\psi_{I c e, L}^{w 2 i}$ and $\psi_{I c e, R}^{w 2 i}$, $\psi_{I c e, L}^{i 2 w}$ and $\psi_{I c e, R}^{i 2 w}$ have also been solved by a variety of methods, e.g., by the Wiener-Hopf method ${ }^{14}$ and by the matched eigenfunction expansion method. ${ }^{9}$

At infinity, there will only be the travelling wave and the velocity potentials above have the following asymptotic forms

$$
\begin{gathered}
\psi_{k}^{r}=A_{k}^{ \pm} \mathrm{e}^{\mp k_{0} x} \frac{\cos \left[k_{0}(z+H)\right]}{\cos \left(k_{0} H\right)} \text { as } x \rightarrow \pm \infty(k=1,2,3), \\
\psi_{0}^{s+}=\left(\mathrm{e}^{+k_{0} x}+r_{0}^{+} \mathrm{e}^{-k_{0} x}\right) \frac{\cos \left[k_{0}(z+H)\right]}{\cos \left(k_{0} H\right)} \text { as } x \rightarrow+\infty, \\
\psi_{0}^{s+}=t_{0}^{+} \mathrm{e}^{+k_{0} x} \frac{\cos \left[k_{0}(z+H)\right]}{\cos \left(k_{0} H\right)} \text { as } x \rightarrow-\infty, \\
\psi_{0}^{s-}=t_{0}^{-} \mathrm{e}^{-k_{0} x} \frac{\cos \left[k_{0}(z+H)\right]}{\cos \left(k_{0} H\right)} \text { as } x \rightarrow+\infty, \\
\psi_{0}^{s-}=\left(\mathrm{e}^{-k_{0} x}+r_{0}^{-} \mathrm{e}^{+k_{0} x}\right) \frac{\cos \left[k_{0}(z+H)\right]}{\cos \left(k_{0} H\right)} \text { as } x \rightarrow-\infty, \\
\psi_{I c e, L}^{w 2 i}=\left(\mathrm{e}^{+k_{0} x}+R_{L, 0}^{w 2 i} \mathrm{e}^{-k_{0} x}\right) \frac{\cos \left[k_{0}(z+H)\right]}{\cos \left(k_{0} H\right)} \text { as } x \rightarrow+\infty, \\
\psi_{I c e, L}^{w 2 i}=T_{L, 0}^{w 2 i} \mathrm{e}^{+\kappa_{0}^{(1)} x} \frac{\cos \left[\kappa_{0}^{(1)}(z+H)\right]}{\cos \left[\kappa_{0}^{(1)}\left(H-d_{1}\right)\right]} \text { as } x \rightarrow-\infty, \\
\psi_{I c e, R}^{w 2 i}=T_{R, 0}^{w 2 i} \mathrm{e}^{-\kappa_{0}^{(2)} x} \frac{\cos \left[\kappa_{0}^{(2)}(z+H)\right]}{\cos \left[\kappa_{0}^{(2)}\left(H-d_{2}\right)\right]} \text { as } x \rightarrow+\infty,
\end{gathered}
$$

$$
\psi_{I c e, R}^{w 2 i}=\left(\mathrm{e}^{-k_{0} x}+R_{R, 0}^{w 2 i} \mathrm{e}^{+k_{0} x}\right) \frac{\cos \left[k_{0}(z+H)\right]}{\cos \left(k_{0} H\right)} \text { as } x \rightarrow-\infty,
$$

$$
\begin{gathered}
\psi_{I c e, L}^{i 2 w}=T_{L, 0}^{i 2 w} \mathrm{e}^{-k_{0} x} \frac{\cos \left[k_{0}(z+H)\right]}{\cos \left(k_{0} H\right)} \text { as } x \rightarrow+\infty, \\
\psi_{I c e, L}^{i 2 w}=\left(\mathrm{e}^{-\kappa_{0}^{(1)} x}+R_{L, 0}^{i 2 w} \mathrm{e}^{+\kappa_{0}^{(1)} x}\right) \frac{\cos \left[\kappa_{0}^{(1)}(z+H)\right]}{\cos \left[\kappa_{0}^{(1)}\left(H-d_{1}\right)\right]} \text { as } x \rightarrow-\infty,
\end{gathered}
$$

where $A_{k}^{ \pm}$is the amplitude of the radiation potential at $x \rightarrow \pm \infty$ due to the forced body oscillation in the $k$-th mode with unit amplitude; $r_{0}^{ \pm}$and $t_{0}^{ \pm}$are, respectively, the reflection and transmission coefficients for the incident wave propagating across the fixed body; $R_{L, 0}^{w 2 i}$ and $R_{R, 0}^{w 2 i}, T_{L, 0}^{w 2 i}$ and $T_{R, 0}^{w 2 i}$ are, respectively, the reflection and transmission coefficients for the incident wave propagating from the open water to the ice covered region; $R_{L, 0}^{i 2 w}$ and $R_{R, 0}^{i 2 w}, T_{L, 0}^{i 2 w}$ and $T_{R, 0}^{i 2 w}$ are, respectively, the reflection and transmission coefficients for the incident wave propagating from the ice covered region to the open water.

With these solutions for the pure wave/body and pure wave/ice interaction problems, we are now able to study the radiation and scattering problems of the body floating on a wide polynya, or the wave/ice/body interaction problem, following the procedure in the work of Srokosz and Evans ${ }^{28}$ for the wide spacing multibodies/wave interaction problem.

\section{Radiation potential}

Here we consider a floating body located at $x=0$ undergoing oscillation in the $k$ th mode. Near the floating body, we may write the velocity potential as

$$
\phi_{k}(x, z)=\psi_{k}^{r}(x, z)+\varepsilon_{k}^{1} \psi_{0}^{s+}(x, z)+\varepsilon_{k}^{2} \psi_{0}^{s-}(x, z)
$$

Similarly for the ice sheets, noticing that edges are located at $x=x_{j}(j=1,2)$, we may write the velocity potential as

$$
\phi_{k}(x, z)=\eta_{k}^{1} \psi_{I c e, L}^{w 2 i}\left(x-x_{1}, z\right)
$$

for the left ice sheet and

$$
\phi_{k}(x, z)=\eta_{k}^{2} \psi_{I c e, R}^{w 2 i}\left(x-x_{2}, z\right),
$$

for the right ice sheet.

Here $\varepsilon_{k}^{1}$ and $\varepsilon_{k}^{2}, \eta_{k}^{1}$ and $\eta_{k}^{2}$ are unknown coefficients. $x-x_{1}$ and $x-x_{2}$ are used, respectively, in Eqs. (31) and (32) instead of using $x$, since the ice sheet edge is not situated at the origin $x=0$, whereas the solutions $\psi_{I c e, L}^{w 2 i}$ and $\psi_{I c e, R}^{w 2 i}$ correspond to edge being at the origin.

We may notice that the first term on the right-hand side of Eq. (30) is the solution in the absence of the semi-infinite ice sheets. The second and third terms represent the scattering of the wave reflected back from the ice sheets, which are given by Eqs. (20)-(23) based on the large gap assumption. In Eqs. (31) and (32), the right-hand sides represent the interaction of the propagating wave with the semi-infinite ice sheet, with the incident potential due to the radiated wave of the body.

To determine the unknown coefficients, we choose two vertical interfaces located at $x=x_{L}$ and $x=x_{R}$, respectively, as shown in Fig. 1. They are assumed to be sufficiently away 
from the body and ice edge and the asymptotic formulas in Eqs. (19)-(29) apply. On these two vertical interfaces, the continuous condition of pressure and normal velocity should be enforced, i.e.,

$\phi_{k}\left(x_{L^{-}}, z\right)=\phi_{k}\left(x_{L+}, z\right), \quad \frac{\partial \phi_{k}\left(x_{L^{-}}, z\right)}{\partial x}=\frac{\partial \phi_{k}\left(x_{L+}, z\right)}{\partial x}$

and

$\phi_{k}\left(x_{R-}, z\right)=\phi_{k}\left(x_{R+}, z\right), \quad \frac{\partial \phi_{k}\left(x_{R-}, z\right)}{\partial x}=\frac{\partial \phi_{k}\left(x_{R+}, z\right)}{\partial x}$,

where the subscripts + and - mean that the potentials should be taken from the solutions on the left- and right-hand sides, respectively. Substituting the potentials on each side of the interfaces into the above equations, we can have

$$
\begin{gathered}
\varepsilon_{k}^{1}=-\left[\left(A_{k}^{-} t_{0}^{-}-A_{k}^{+} r_{0}^{-}\right) R_{L, 0}^{w 2 i} R_{R, 0}^{w 2 i} \mathrm{e}^{k_{0}\left(x_{1}-x_{2}\right)}\right. \\
\left.+A_{k}^{+} R_{R, 0}^{w 2 i} \mathrm{e}^{-k_{0}\left(x_{1}+x_{2}\right)}\right] / M, \\
\varepsilon_{k}^{2}=-\left[\left(A_{k}^{+} t_{0}^{+}-A_{k}^{-} r_{0}^{+}\right) R_{L, 0}^{w 2 i} R_{R, 0}^{w 2 i} \mathrm{e}^{k_{0}\left(x_{1}-x_{2}\right)}\right. \\
\left.+A_{k}^{-} R_{L, 0}^{w 2 i} \mathrm{e}^{k_{0}\left(x_{1}+x_{2}\right)}\right] / M, \\
\eta_{k}^{1}=-\left[\left(A_{k}^{+} t_{0}^{+}-A_{k}^{-} r_{0}^{+}\right) R_{R, 0}^{w 2 i} \mathrm{e}^{-k_{0} x_{2}}+A_{k}^{-} \mathrm{e}^{k_{0} x_{2}}\right] / M, \\
\eta_{k}^{2}=-\left[\left(A_{k}^{-} t_{0}^{-}-A_{k}^{+} r_{0}^{-}\right) R_{L, 0}^{w 2 i} \mathrm{e}^{k_{0} x_{1}}+A_{k}^{+} \mathrm{e}^{-k_{0} x_{1}}\right] / M,
\end{gathered}
$$

where

$$
\begin{aligned}
M= & \left(t_{0}^{+} t_{0}^{-}-r_{0}^{+} r_{0}^{-}\right) R_{L, 0}^{w 2 i} R_{R, 0}^{w 2 i} \mathrm{e}^{k_{0}\left(x_{1}-x_{2}\right)}-\mathrm{e}^{-k_{0}\left(x_{1}-x_{2}\right)} \\
& +r_{0}^{-} R_{L, 0}^{w 2 i} \mathrm{e}^{k_{0}\left(x_{1}+x_{2}\right)}+r_{0}^{+} R_{R, 0}^{w 2 i} \mathrm{e}^{-k_{0}\left(x_{1}+x_{2}\right)} .
\end{aligned}
$$

Invoking Eq. (30), we can obtain the added mass and damping coefficient for the body floating on a polynya from the results for open water or

$$
\mu_{k j}-i \frac{\lambda_{k j}}{\omega}=\mu_{k j}^{o}-i \frac{\lambda_{k j}^{o}}{\omega}-\varepsilon_{j}^{1} \frac{f_{E, k}^{o+}}{g}-\varepsilon_{j}^{2} \frac{f_{E, k}^{o-}}{g},
$$

where the superscript o means that the results are from open water, and + and - in $f_{E, k}^{o}$ mean that the wave exciting force is due to the incident wave opposite to and along the $x$-axis, respectively. Here the incident wave potential in Eq. (40) is the same as that defined after Eq. (1) with zero ice thickness when computing $f_{E, k}^{o \pm}$.

From Eqs. (31) and (32), together with Eqs. (25) and (26), we can obtain the asymptotic expressions for the velocity potential $\phi_{k}$,

$$
\begin{aligned}
& \phi_{k}(x, z)=\eta_{k}^{1} T_{L, 0}^{w 2 i} \mathrm{e}^{+\kappa_{0}^{(1)}\left(x-x_{1}\right)} \frac{\cos \left[\kappa_{0}^{(1)}(z+H)\right]}{\cos \left[\kappa_{0}^{(1)}\left(H-d_{1}\right)\right]} \text { as } x \rightarrow-\infty, \\
& \phi_{k}(x, z)=\eta_{k}^{2} T_{R, 0}^{w 2 i} \mathrm{e}^{-\kappa_{0}^{(2)}\left(x-x_{2}\right)} \frac{\cos \left[\kappa_{0}^{(2)}(z+H)\right]}{\cos \left[\kappa_{0}^{(2)}\left(H-d_{2}\right)\right]} \text { as } x \rightarrow+\infty .
\end{aligned}
$$

Substituting these into the far field formula of Ren et al. ${ }^{25}$ the damping coefficient can be also written as

$\lambda_{k j}=\rho \omega\left[Q_{0}^{(1)} C_{g}^{(1)}\left(\eta_{j}^{1}\right)\left(\eta_{k}^{1}\right)^{*}\left|T_{L, 0}^{w 2 i}\right|^{2}+Q_{0}^{(2)} C_{g}^{(2)}\left(\eta_{j}^{2}\right)\left(\eta_{k}^{2}\right)^{*}\left|T_{R, 0}^{w 2 i}\right|^{2}\right]$

$$
(k, j=1,2,3),
$$

where the superscript $*$ denotes complex conjugation,

$$
Q_{0}^{(j)}=\frac{\rho \omega\left[L_{j}\left(\kappa_{0}^{(j)}\right)^{4}+\rho g\right]}{\left[L_{j}\left(\kappa_{0}^{(j)}\right)^{4}+\rho g-m_{j} \omega^{2}\right]^{2}} \quad(j=1,2),
$$

and

$$
C_{g}^{(j)}=i \frac{\frac{\omega}{2 \kappa_{0}^{(j)}}\left(1+\frac{2 \kappa_{0}^{(j)}\left(H-d_{j}\right)}{\sin \left[2 \kappa_{0}^{(j)}\left(H-d_{j}\right)\right]}\right)+\frac{2 L_{j}\left(\kappa_{0}^{(j)}\right)^{3} \omega}{L_{j}\left(\kappa_{0}^{(j)}\right)^{4}+\rho g-m_{j} \omega^{2}}}{\frac{L_{j}\left(\kappa_{0}^{(j)}\right)^{4}+\rho g}{L_{j}\left(\kappa_{0}^{(j)}\right)^{4}+\rho g-m_{j} \omega^{2}}}(j=1,2)
$$

is the wave group velocity in the ice covered region.

\section{Scattering potential}

We follow the procedure similar to that used for the radiation above. Near the body, we may write the velocity potential as

$$
\phi_{0}(x, z)=\gamma_{1} \psi_{0}^{s+}(x, z)+\gamma_{2} \psi_{0}^{s-}(x, z),
$$

and near the left and right ice sheets, we may write the velocity potential, respectively, as

$$
\begin{gathered}
\phi_{0}(x, z)=\psi_{\text {Ice }, L}^{i 2 w}\left(x-x_{1}, z\right)+\beta_{1} \psi_{\text {Ice }, L}^{w 2 i}\left(x-x_{1}, z\right), \\
\phi_{0}(x, z)=\beta_{2} \psi_{\text {Ice }, R}^{w 2 i}\left(x-x_{2}, z\right),
\end{gathered}
$$

where $\gamma_{1}$ and $\gamma_{2}, \beta_{1}$ and $\beta_{2}$ are constants to be found. The above two equations are based on the assumption that the incident wave is propagating beneath the left ice sheet along the positive direction of the $x$-axis. Substituting Eqs. (19)-(29) into Eqs. (46)-(48) and imposing the matching conditions on $x=x_{L}$ and on $x=x_{R}$, we have

$$
\begin{gathered}
\gamma_{1}=-t_{0}^{-} R_{R, 0}^{w 2 i} T_{L, 0}^{i 2 w} \mathrm{e}^{-k_{0} x_{2}} / N, \\
\gamma_{2}=-\left(\mathrm{e}^{+k_{0} x_{2}}-r_{0}^{+} R_{R, 0}^{w 2 i} \mathrm{e}^{-k_{0} x_{2}}\right) T_{L, 0}^{i 2 w} / N,
\end{gathered}
$$

$\beta_{1}=\left[-r_{0}^{-} \mathrm{e}^{k_{0} x_{2}}+\left(r_{0}^{-} r_{0}^{+}-t_{0}^{-} t_{0}^{+}\right) R_{R, 0}^{w 2 i} \mathrm{e}^{-k_{0} x_{2}}\right] T_{L, 0}^{i 2 w} \mathrm{e}^{+k_{0} x_{1}} / N$,

$$
\beta_{2}=-t_{0}^{-} T_{L, 0}^{i 2 w} / N
$$

where

$$
\begin{aligned}
N= & \left(t_{0}^{-} t_{0}^{+}-r_{0}^{-} r_{0}^{+}\right) R_{L, 0}^{w 2 i} R_{R, 0}^{w 2 i} \mathrm{e}^{k_{0}\left(x_{1}-x_{2}\right)}-\mathrm{e}^{-k_{0}\left(x_{1}-x_{2}\right)} \\
& +r_{0}^{-} R_{L, 0}^{w 2 i} \mathrm{e}^{+k_{0}\left(x_{1}+x_{2}\right)}+r_{0}^{+} R_{R, 0}^{w 2 i} \mathrm{e}^{-k_{0}\left(x_{1}+x_{2}\right)}
\end{aligned}
$$

Invoking Eq. (46) we can obtain the wave exciting force for the body floating on polynya from the results for open water or

$$
f_{E, k}=\gamma_{1} f_{E, k}^{o+}+\gamma_{2} f_{E, k}^{o-}
$$

when Eqs. (12) and (46) are used.

At infinity, the asymptotic form of the velocity potential can be written as 


$$
\phi(x, z)=\left\{\begin{array}{cc}
\left(\mathrm{e}^{-\kappa_{0}^{(1)}\left(x-x_{1}\right)}+\mathrm{Re}^{\kappa_{0}^{(1)}\left(x-x_{1}\right)}\right) \frac{\cos \left[\kappa_{0}^{(1)}(z+H)\right]}{\cos \left[\kappa_{0}^{(1)}\left(H-d_{1}\right)\right]}, & x \rightarrow-\infty \\
\operatorname{Te}^{-\kappa_{0}^{(2)}\left(x-x_{2}\right)} \frac{\cos \left[\kappa_{0}^{(2)}(z+H)\right]}{\cos \left[\kappa_{0}^{(2)}\left(H-d_{2}\right)\right]}, & x \rightarrow+\infty
\end{array},\right.
$$

where $\mathrm{R}$ and $\mathrm{T}$ are the reflection and transmission coefficients, respectively. Then from Eqs. (47) and (48), together with Eqs. (29), (25), and (26), respectively, we have

$$
\begin{gathered}
\mathrm{R}=R_{L, 0}^{i 2 w}+\beta_{1} T_{L, 0}^{w 2 i}, \\
\mathrm{~T}=\beta_{2} T_{R, 0}^{w 2 i} .
\end{gathered}
$$

Similar to the damping coefficient, through the asymptotic expressions of Eqs. (55), (41), and (42), the far field equation for the wave exciting force (e.g., Ren et al. ${ }^{25}$ ) gives

$$
\begin{aligned}
& f_{E, k}^{-}=-2 \mathrm{i} \rho g \eta_{k}^{1} T_{L, 0}^{w 2 i} C_{g}^{(1)} Q_{0}^{(1)} \\
& f_{E, k}^{+}=-2 \mathrm{i} \rho g \eta_{k}^{2} T_{R, 0}^{w 2 i} C_{g}^{(2)} Q_{0}^{(2)}
\end{aligned}(k=1,2,3),
$$

where $Q_{0}^{(j)}$ and $C_{g}^{(j)}$ are defined in Eqs. (44) and (45), respectively. Invoking Eqs. (43) and (58), we can obtain the link between the damping coefficient and exciting force as

$$
\begin{gathered}
\lambda_{k j}=-\frac{\omega}{4 \rho g^{2}}\left[\frac{1}{C_{g}^{(1)} Q_{0}^{(1)}}\left(f_{E, j}^{-}\right)\left(f_{E, k}^{-}\right)^{*}+\frac{1}{C_{g}^{(2)} Q_{0}^{(2)}}\left(f_{E, j}^{+}\right)\left(f_{E, k}^{+}\right)^{*}\right] \\
(k, j=1,2,3) .
\end{gathered}
$$

It is noticeable that the identity above is the same as Eq. (47) in the work of $\mathrm{Li}$ et al. ${ }^{32}$ obtained from the exact solution.

\section{NUMERICAL RESULTS}

We shall first demonstrate the accuracy and efficiency of the present method. This is to be achieved by comparing the obtained results with the "exact" solution. After the method is verified, we shall use the formulation to provide some insights into the features of the hydrodynamic force and body motion, in particular its highly oscillatory behaviour. The numerical results are presented in the nondimensionalized form, based on a characteristic length scale, the density of water $\rho$, and acceleration due to gravity $g$.

\section{A. Wave propagation across a polynya}

We first consider the case for a wave propagating underneath the left ice sheet. It passes through a polynya and moves into the right ice sheet. As the body is removed, the wide polynya approximation is made on the basis that its width is much larger than the wavelength. Then the reflection and transmission coefficients can be obtained directly by letting $r_{0}^{+}=r_{0}^{-}=0, t_{0}^{+}=t_{0}^{-}=1$ in Eqs. (56) and (57) or

$$
\begin{gathered}
\mathrm{R}=R_{L, 0}^{i 2 w}+\frac{R_{R, 0}^{w 2 i} T_{L, 0}^{i 2 w} T_{L, 0}^{w 2 i} \mathrm{e}^{-2 k_{0}\left(x_{2}-x_{1}\right)}}{1-R_{L, 0}^{w 2 i} R_{R, 0}^{w 2 i} \mathrm{e}^{-2 k_{0}\left(x_{2}-x_{1}\right)}}, \\
\mathrm{T}=\frac{T_{L, 0}^{i 2 w} T_{R, 0}^{w 2 i} \mathrm{e}^{-k_{0}\left(x_{2}-x_{1}\right)}}{1-R_{L, 0}^{w 2 i} R_{R, 0}^{w 2 i} \mathrm{e}^{-2 k_{0}\left(x_{2}-x_{1}\right)}},
\end{gathered}
$$

which can be found to satisfy the energy conservation equation [i.e., Eq. (A2) in the work of Ren et al. ${ }^{25}$ ]. These two equations may also be obtained by using the procedure in the work of Meylan and Squire ${ }^{33}$ for wave propagation across a finite floe. Assume that the incoming wave is from $x=-\infty$. Near the left ice sheet edge at $x=x_{1}$, if we ignore the evanescent waves, we can consider the two travelling waves with complex amplitudes $w_{a}$ and $w_{b}$, propagating along and opposite to the $x$-axis, respectively. The reflection coefficient $\mathrm{R}$ at $x=-\infty$ should be due to the reflection (semi-infinite ice to semi-infinite free surface) and transmission of $w_{b}$ (semi-infinite water to semi-infinite ice). Thus

$$
\mathrm{R}=R_{L, 0}^{i 2 w}+w_{b} T_{L, 0}^{w 2 i}
$$

On the other hand, $w_{a}$ is due to the combination of transmission (semi-infinite ice to semi-infinite water) and reflection of $w_{b}$ (semi-infinite water to semi-infinite ice),

$$
w_{a}=T_{L, 0}^{i 2 w}+w_{b} R_{L, 0}^{w 2 i} .
$$

At the other ice sheet edge $x=x_{2}$, the waves of $w_{a}$ and $w_{b}$ should have a phase shift

$$
\begin{gathered}
w_{a} \mathrm{e}^{-k_{0}\left(x-x_{1}\right)}=w_{a} \mathrm{e}^{-k_{0}\left(x_{2}-x_{1}\right)} \mathrm{e}^{-k_{0}\left(x-x_{2}\right)}, \\
w_{b} \mathrm{e}^{k_{0}\left(x-x_{1}\right)}=w_{b} \mathrm{e}^{k_{0}\left(x_{2}-x_{1}\right)} \mathrm{e}^{k_{0}\left(x-x_{2}\right)},
\end{gathered}
$$

which means that their complex amplitudes at $x=x_{2}$ become $w_{a} \mathrm{e}^{-k_{0}\left(x_{2}-x_{1}\right)}$ and $w_{b} \mathrm{e}^{k_{0}\left(x_{2}-x_{1}\right)}$, respectively. Then $w_{b} \mathrm{e}^{k_{0}\left(x_{2}-x_{1}\right)}$ is due to the reflection of $w_{a} \mathrm{e}^{-k_{0}\left(x_{2}-x_{1}\right)}$ (semi-infinite water to semi-infinite ice),

$$
w_{b} \mathrm{e}^{k_{0}\left(x_{2}-x_{1}\right)}=w_{a} \mathrm{e}^{-k_{0}\left(x_{2}-x_{1}\right)} R_{R, 0}^{w 2 i},
$$

and the wave at $x=+\infty$ is due to the transmission of $w_{a} \mathrm{e}^{-k_{0}\left(x_{2}-x_{1}\right)}$,

$$
\mathrm{T}=w_{a} \mathrm{e}^{-k_{0}\left(x_{2}-x_{1}\right)} T_{R, 0}^{w 2 i} .
$$

From Eqs. (63) and (66), we have

$$
\begin{gathered}
w_{a}=\frac{T_{L, 0}^{i 2 w}}{1-R_{R, 0}^{w 2 i} R_{L, 0}^{w 2 i} \mathrm{e}^{-2 k_{0}\left(x_{2}-x_{1}\right)},} \\
w_{b}=\frac{T_{L, 0}^{i 2 w} R_{R, 0}^{w 2 i} \mathrm{e}^{-2 k_{0}\left(x_{2}-x_{1}\right)}}{1-R_{R, 0}^{w 2 i} R_{L, 0}^{w 2 i} \mathrm{e}^{-2 k_{0}\left(x_{2}-x_{1}\right)} .}
\end{gathered}
$$

Substituting Eqs. (69) and (68) into Eqs. (62) and (67), we can further obtain the expression for $\mathrm{R}$ and $\mathrm{T}$, which are identical to Eqs. (60) and (61). Therefore for this particular case, the procedure in the work of Meylan and Squire ${ }^{33}$ and the present method give the same result. It also ought to point out that in the work of Meylan and Squire, ${ }^{33}$ the origin was taken at one of the edges, while here in Eqs. (47) and (48), the origin is taken at $x=x_{1}$ and $x_{2}$, respectively.

The reflection and transmission coefficients between semi-infinite $i 2 w$ (ice to water) and semi-infinite $w 2 i$ (water 


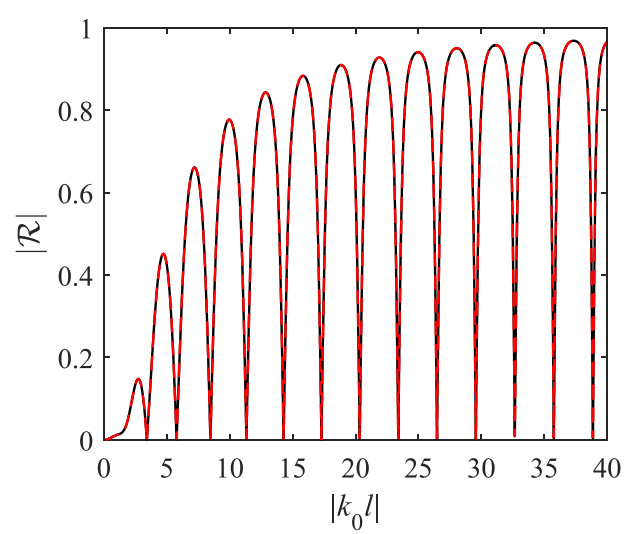

(a)

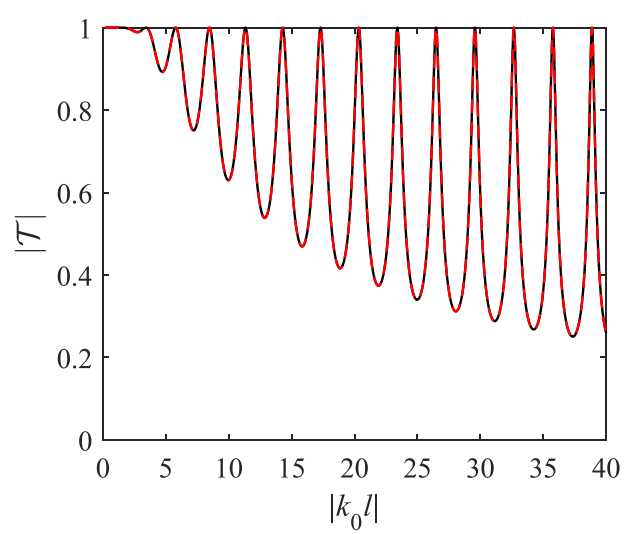

(b)

FIG. 2. Reflection and transmission coefficients for a wave propagating across a polynya with zero ice draught. (a) Reflection coefficient; (b) transmission coefficient. Solid lines: exact results computed by the matched eigenfunction expansions in the work of Ren et al. ${ }^{25}$ dashed lines: computed by formula Eqs. (60) and (61) $\left(H=5, x_{1}=-x_{2}=-0.5, h_{2}=h_{1}=0.02, d_{2}=d_{1}=0, m_{2}=m_{1}=0.018\right.$, and $\left.L_{2}=L_{1}=0.003647\right)$.

to ice) are in fact related. Similar to Meylan and Squire, ${ }^{33}$ we may use Stokes time reverse and obtain

$$
R_{L, 0}^{i 2 w}=-\frac{\left(R_{L, 0}^{w 2 i}\right)^{*} T_{L, 0}^{w 2 i}}{\left(T_{L, 0}^{w 2 i}\right)^{*}}, \quad T_{L, 0}^{i 2 w}=\frac{1-\left|R_{L, 0}^{w 2 i}\right|^{2}}{\left(T_{L, 0}^{w 2 i}\right)^{*}},
$$

which can also be obtained by using the Green identity, for example, Eq. (A1) in the work of Ren et al. ${ }^{25}$ through replacing $\phi$ and $\phi^{*}$ by $\phi^{i 2 w}$ and $\left(\phi^{w 2 i}\right)^{*}$, then by $\phi^{i 2 w}$ and $\phi^{w 2 i}$, and by $\phi^{w 2 i}$ and $\left(\phi^{w 2 i}\right)^{*}$.

To verify the accuracy and efficiency of the present method, we consider the polynya with the following parameters:

$$
\begin{aligned}
& H=5, \quad h_{2}=h_{1}=0.02, \quad m_{2}=m_{1}=0.018 \\
& L_{2}=L_{1}=0.003647 .
\end{aligned}
$$

The characteristic length scale above has been chosen as the polynya width $l=x_{2}-x_{1}$. Figures 2 and 3 show the reflection and transmission coefficients at zero ice draught and at $d_{2}=d_{1}=0.018$, respectively, against $\left|k_{0} l\right|$. It can be seen from these two figures that the present numerical results agree very well with those exact solutions which are calculated through the eigenfunction method in the work of Ren et al. ${ }^{25}$ Strictly speaking, the present approximation should be valid only when the width is much larger than the wavelength or $\left|k_{0} l\right| \gg 1$. However, it can be seen from Eqs. (60) and (61) that $|\mathrm{R}| \rightarrow 0$ and $|\mathrm{T}| \rightarrow 1$ when $\left|k_{0} l\right| \rightarrow 0$, which is in fact a result of the exact solution. ${ }^{16}$ Thus it is not a total surprise that the present approximate method can give such an accurate result for the whole wave frequency span shown in Figs. 2 and 3.

From the exact solution of Chung and Linton ${ }^{16}$ for zero ice draught, it was found that there were an infinite number of discrete frequencies at which the reflection coefficient $\mathrm{R}$ could be zero. Here when the left and right ice sheets have the same physical properties, we have $R=R_{R, 0}^{w 2 i}$ $=R_{L, 0}^{w 2 i}, T=T_{R, 0}^{w 2 i}=T_{L, 0}^{w 2 i}$. Substitution of Eq. (70) into Eq. (60) provides

$$
\mathrm{R}=-\frac{T}{T^{*} R} S_{\mathrm{R}}(\omega),
$$

where

$$
S_{\mathrm{R}}(\omega)=\frac{\mathrm{e}^{2 \mathrm{i}(\delta+\beta)}-1}{\mathrm{e}^{2 \mathrm{i}(\delta+\beta)}-1 /|R|^{2}},
$$

with $\delta=\mathrm{i} k_{0} l$ and $\beta=\operatorname{Arg}(R) \in[0,2 \pi)$ which is the argument of $R$. Equation (73) maps the unit circle $\mathrm{e}^{2 \mathrm{i}(\delta+\beta)}$ to a circle with the centre at $1 /\left(1+1 /|R|^{2}\right)$ and a radius of $1 /\left(1+1 /|R|^{2}\right)$, from which we find that $|\mathrm{R}|$ will reach its troughs (i.e., zero) when $\delta$ equals

$$
\delta_{T}^{\mathrm{R}}=n \pi-\beta,
$$

and will reach its peaks when $\delta$ equals

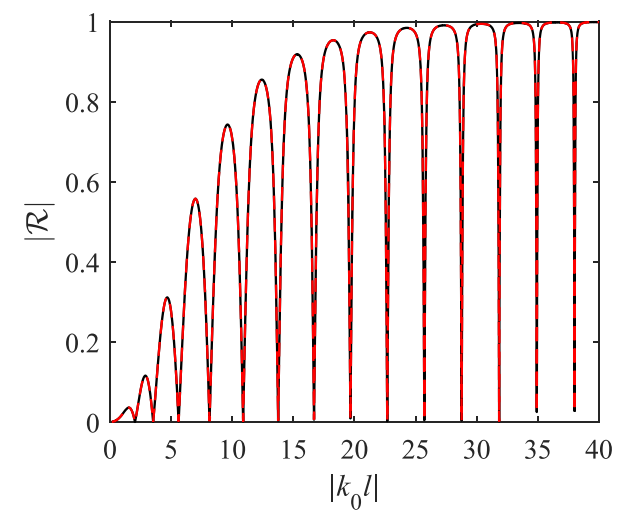

(a)

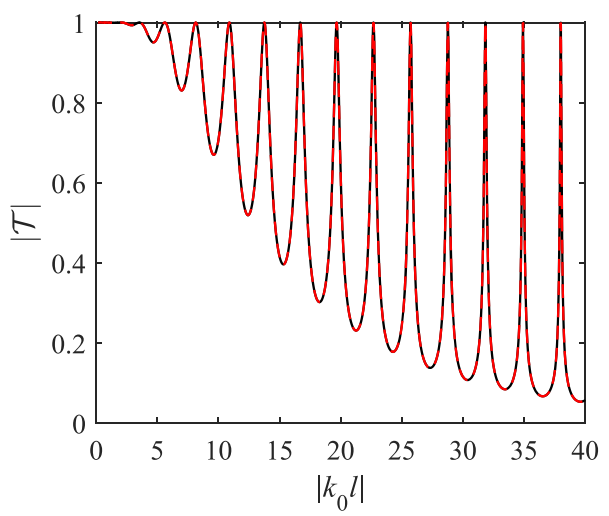

(b)
FIG. 3. Reflection and transmission coefficients for a wave propagating across a polynya with nonzero ice draught. (a) Reflection coefficient; (b) transmission coefficient. Solid lines: exact results computed by the matched eigenfunction expansions in the work of Ren et al. ${ }^{25}$ dashed lines: computed by formula Eqs. (60) and (61) $(H=5$, $x_{1}=-x_{2}=-0.5, h_{2}=h_{1}=0.02, d_{2}=d_{1}$ $=0.018, m_{2}=m_{1}=0.018$, and $L_{2}=L_{1}$ $=0.003$ 647). 


$$
\delta_{P}^{\mathrm{R}}=n \pi+\pi / 2-\beta,
$$

where $n$ includes all integers which ensure $\delta<0$ required based on the definition of $k_{0}$. This can be seen in Fig. 2 at zero ice draught. These two equations can be further explained by the physical process of the wave motion in the polynya. From Eq. (70), the phase of the first term on the right-hand side of Eq. (62) can be obtained as

$$
\operatorname{Arg}\left(R_{L, 0}^{i 2 w}\right)=-\beta+2 \gamma+\pi,
$$

where $\gamma=\operatorname{Arg}(T)$. When the incoming wave passes through the ice sheet edge at $x=x_{1}$, the transmitted wave can be written as

$$
w_{1}=T_{L, 0}^{i 2 w} \mathrm{e}^{-k_{0}\left(x-x_{1}\right)} .
$$

When $w_{1}$ reaches the right ice sheet edge at $x=x_{2}$, there will be a reflected wave

$$
w_{2}=T_{L, 0}^{i 2 w} R \mathrm{e}^{-k_{0} l} \mathrm{e}^{k_{0}\left(x-x_{2}\right)} .
$$

When $w_{2}$ reaches the left ice sheet edge at $x=x_{1}$, there will be a transmitted wave into the ice sheet,

$$
w_{3}=T_{L, 0}^{i 2 w} R T \mathrm{e}^{-2 k_{0} l} \mathrm{e}^{\kappa_{0}^{(1)}\left(x-x_{1}\right)} .
$$

The phase of the complex amplitude of this wave is then equal to $\beta+2 \gamma+2 \delta$ [note that we have $\operatorname{Arg}\left(T_{L, 0}^{i 2 w}\right)=\operatorname{Arg}\left(T_{L, 0}^{w 2 i}\right)$ according to the second equation in Eq. (70)]. Its difference with the phase in Eq. (76) is then $2 \beta+2 \delta-\pi$. When this is equal to $2 n \pi+$ $\pi$, Eq. (79) will be out of phase with the reflected wave in the first term of Eq. (62) and the combined wave will be reduced. Similarly when the difference is equal to $2 n \pi$, the combined wave will increase. This is consistent with Eqs. (74) and (75).
In addition to the reflection and transmission coefficients $\mathrm{R}$ and $\mathrm{T}$, we further investigate the accuracy of the present method for the local wave, through the wave elevation in a polynya obtained from

$$
\frac{\eta_{0}}{\alpha_{0}}=-\frac{\mathrm{i} \omega}{g} \phi_{0}(x, 0) .
$$

Three points are chosen and they are, respectively, taken at the edge of the left ice sheet $x=x_{1}$, middle in the open water $x=$ $\left(x_{1}+x_{2}\right) / 2$, and the edge of the right ice sheet $x=x_{2}$. The wave elevations are shown in Fig. 4. It can be seen that the results from the present method almost coincide with the exact solution, which shows that the approximate method can give a very accurate result for the local wave across the frequency range.

\section{B. Wave interaction with a submerged ellipse}

The case chosen for further comparison is an elliptic cylinder defined as $\left(x-x_{0}\right)^{2} / a^{2}+\left(z-z_{0}\right)^{2} / b^{2}=1$, where $a$ and $b$ are its half axes in $x$ and $z$ directions, respectively, and $\left(x_{0}, z_{0}\right)$ is the centre of the cylinder, at which the rotational centre is located or $\left(x^{\prime}, z^{\prime}\right)=\left(x_{0}, z_{0}\right)$. The characteristic length scale is chosen as $a$. The exact solution for this problem has been obtained by the source distribution method ${ }^{24}$ using the Green function satisfying all the boundary conditions apart from that on the body surface. Here we use the hybrid method. ${ }^{27}$ Figures 5 and 6, respectively, show the added mass and damping coefficient against the nondimensional wavenumber in deep open water or $\sigma=a \omega^{2} / g$, while Fig. 7 presents the corresponding wave exciting force. The parameters are chosen as the same as those in the work of Sturova. ${ }^{24}$ These figures

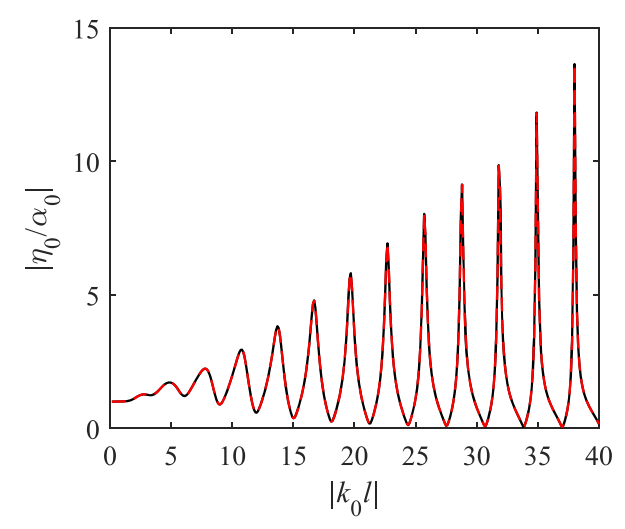

(a)

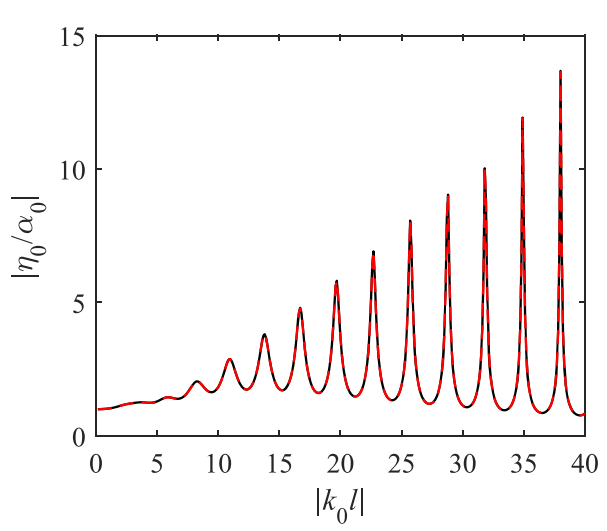

(c)



(b)
FIG. 4. Wave elevation at different location in polynya. (a) $x=x_{1}$. (b) $x=$ $\left(x_{1}+x_{2}\right) / 2$. (c) $x=x_{2}$. Solid lines: exact results computed by the matched eigenfunction expansions in the work of Ren et al. ${ }^{25}$ dashed lines: results computed by formula Eq. (47) for $x<0$ and Eq. (48) for $x>0$. In (b), the dashed line is by Eq. (47), while the dashed-dotted line is by Eq. (48) $\left(H=5, x_{1}=-x_{2}=\right.$ $-0.5, h_{2}=h_{1}=0.02, d_{2}=d_{1}=0.018, m_{2}$ $=m_{1}=0.018$, and $L_{2}=L_{1}=0.003647$ ). 
show that there is no real visible difference between the results obtained from the present method and the exact solution. The damping coefficient and exciting force are also computed by the far field formula and obtained results virtually coincide with those from the near field formula. We may notice that the width of the polynya is only two and half times the body width. The excellent agreement across the frequency span shows the effectiveness of the present method, even though it is based on the large gap assumption.

\section{Wave interaction with a floating rectangle body}

The case chosen now is a floating rectangle body, and its beam $a$ is taken as the characteristic length scale. The added mass and damping coefficient for the floating rectangle body against $\sigma=a \omega^{2} / g$ are, respectively, shown in
Figs. 8 and 9, while the corresponding wave exciting force is presented in Fig. 10. From these figures, we can see that once again there is no visible difference between the results from the present method and the exact solution using the eigenfunction method. ${ }^{25}$ It can be seen from Fig. 3 in the work of Ren et ll $^{25}$ that the radiation force for the body, respectively, floating on the polynya and open water tend to the same value at very small $\sigma$ (noticing that they will be slightly different from that with an ice sheet of non-zero draught). It can also be seen from Fig. 3 that the reflection and transmission coefficients for the wave/semi-infinite ice sheet interaction problem will, respectively, tend to 0 and 1 for very long waves. As $\sigma \rightarrow 0$, from Eq. (40) both coefficients $\varepsilon_{j}^{1}$ and $\varepsilon_{j}^{2}$ will tend to 0 , and from Eq. (54) the coefficients $\gamma_{1}$ and $\gamma_{2}$ will, respectively, tend to 0 and 1 (noticing

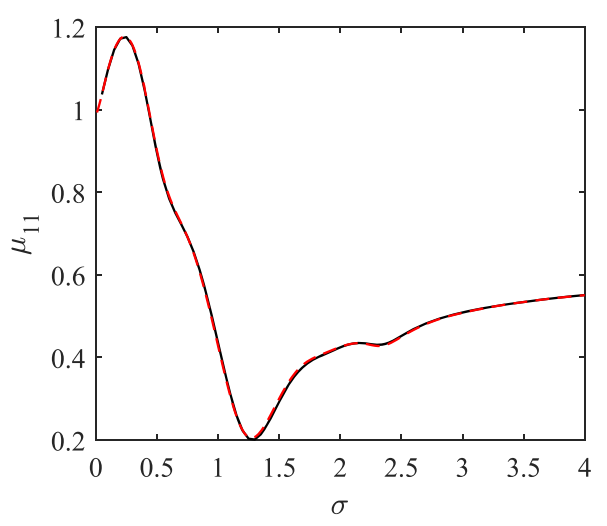

(a)

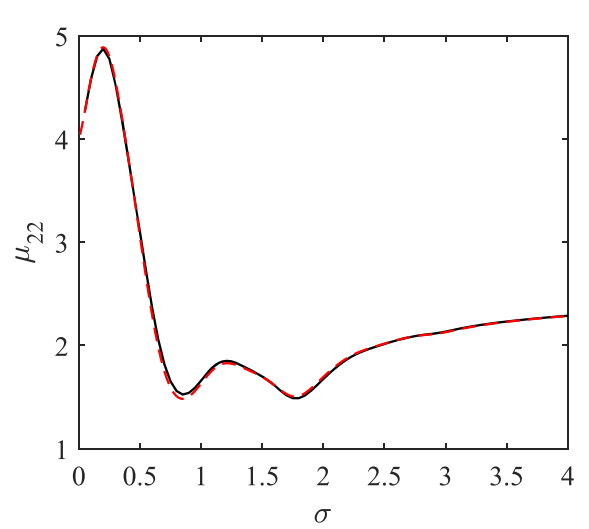

(c)

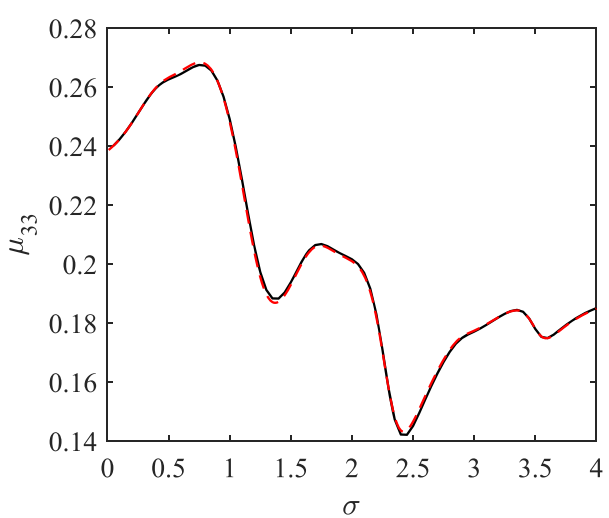

(e)

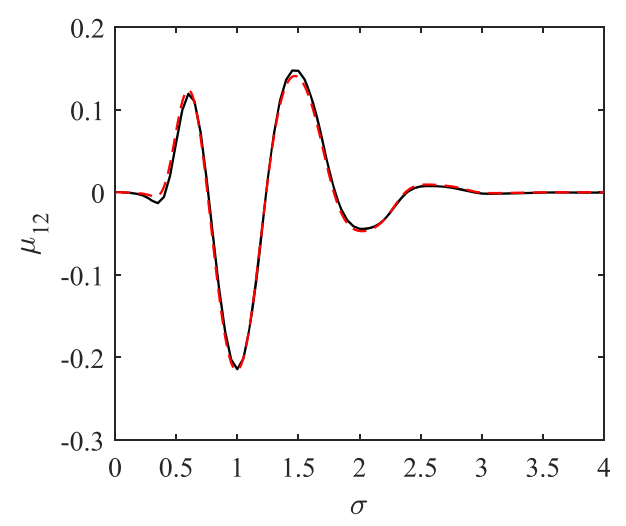

(b)

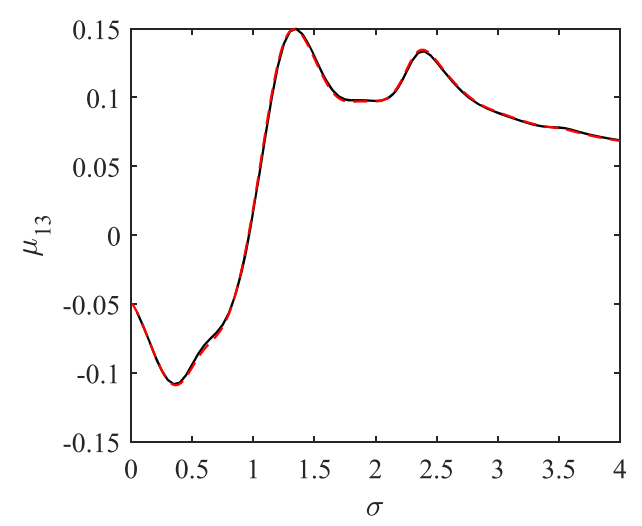

$(d)$

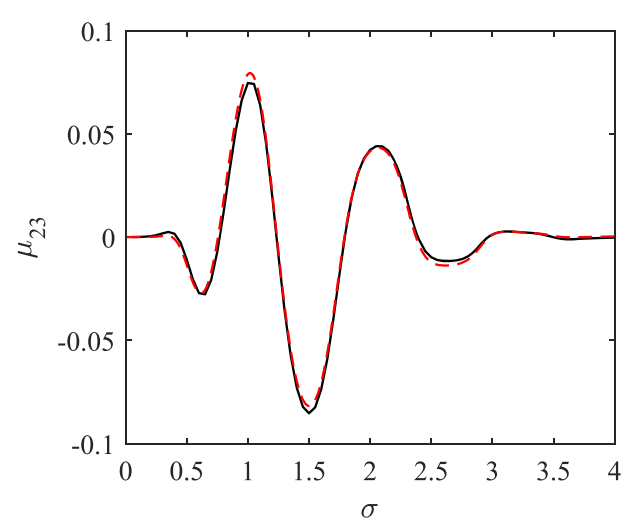

$(f)$
FIG. 5. Added mass of a submerged elliptic cylinder. (a) Sway; (b) swayheave; (c) heave; (d) sway-roll; (e) roll; (f) heave-roll. Solid lines: results computed by the hybrid method in the work of Li et al. ${ }^{27}$ dashed lines: results computed by the present method ( $a=1, b=$ $0.5,\left(x^{\prime}, z^{\prime}\right)=(0,-1), H=25, x_{1}=-x_{2}$ $=-2.5, h_{1}=0.025$ and $h_{2}=0.1, d_{1}=0$ and $d_{2}=0, m_{1}=0.0225$ and $m_{2}=0.09$, and $L_{1}=0.0356$ and $L_{2}=2.2791$ ). 
that there is a phase difference $-k_{0} x_{1}$ in the definition of incident potential when computing the exciting force). These are the same as those from the exact solution. Then the hydrodynamic force computed by the present method will tend to that in open water for a very long wave, i.e., tend to the exact solution with the ice draught effect ignored.

We then investigate the accuracy of the wide spacing approximation through varying the gap width between the ice edge and the body. The heave mode is taken as an example. The added mass and damping coefficient are presented in Fig. 11, against $\ell=\ell_{1}=\ell_{2}$, where $\ell_{1}=\left|x_{1}+a / 2\right|$ and $\ell_{2}=\left|x_{2}-a / 2\right|$. At $\sigma=1.0$, the results are almost the same as those from the exact solution even when the ice edge nearly touches the body. The difference begins to appear when $\ell<1.0$ for the cases of $\sigma=2.0$ and $\sigma=3.0$. We have already discussed previously that the result from the present method tends to the exact solution as $\sigma \rightarrow 0$ for any $\ell$. Also at very high frequencies, all the evanescent modes decay rapidly. These with Fig. 11 show that there will be some noticeable difference between the result of wide spacing approximation and the exact solution only when the gap between the ice edge and body is very small and the frequency is within certain range.

\section{Oscillatory features of the hydrodynamic force and body motion}

The above comparisons show that the present method, based on the wide polynya assumption, is accurate and efficient for a body in a polynya across the frequency span. This allows

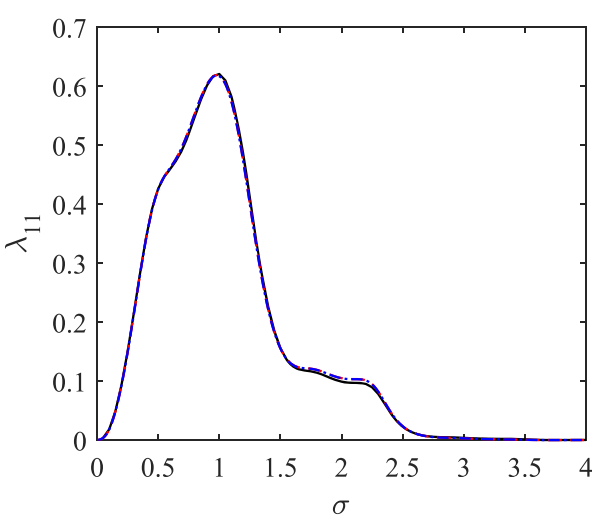

(a)

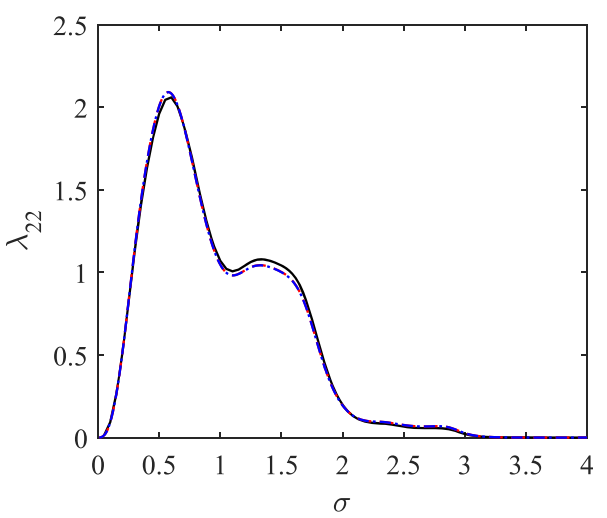

(c)

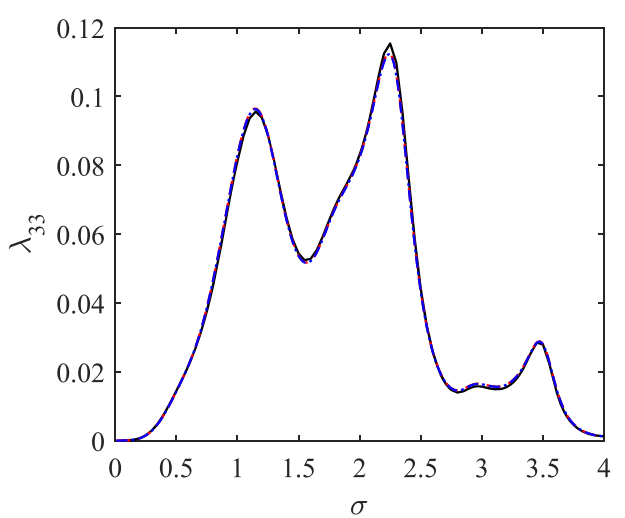

(e)



(b)

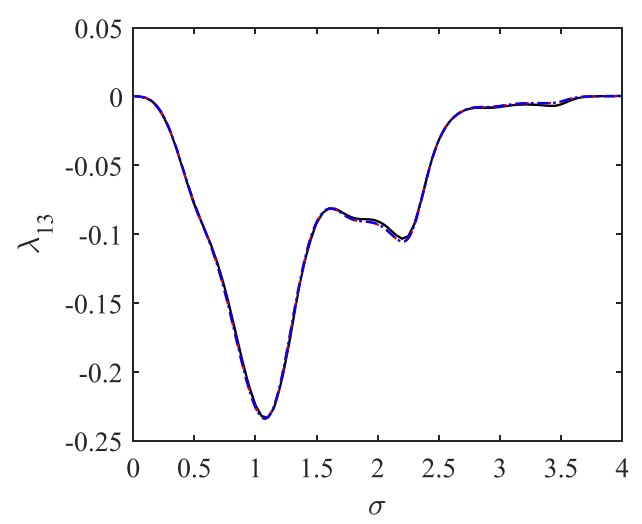

$(d)$

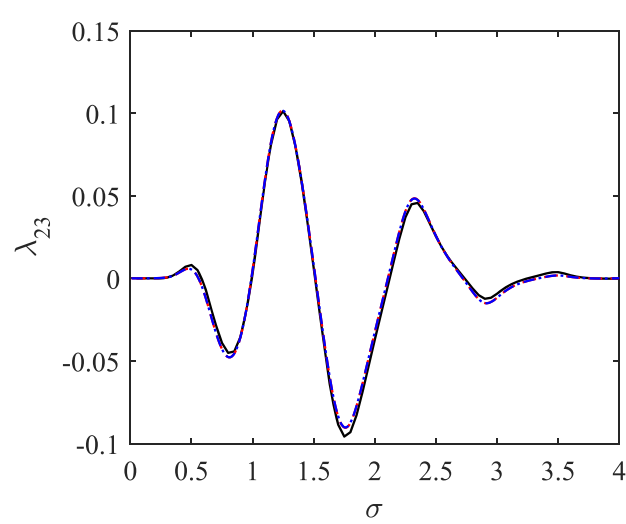

$(f)$
FIG. 6. Damping coefficient of an elliptic cylinder. (a) Sway; (b) swayheave; (c) heave; (d) sway-roll; (e) roll; (f) heave-roll. Solid lines: results computed by the hybrid method in the work of Li et al. $;^{27}$ dashed lines: results computed by the present method; dasheddotted lines: same to dashed lines, but by the far field formula ( $a=1, b=0.5$, $\left(x^{\prime}, z^{\prime}\right)=(0,-1), H=25, x_{1}=-x_{2}=$ $-2.5, h_{1}=0.025$ and $h_{2}=0.1, d_{1}=0$ and $d_{2}=0, m_{1}=0.0225$ and $m_{2}=0.09$, and $L_{1}=0.0356$ and $L_{2}=2.2791$ ). 


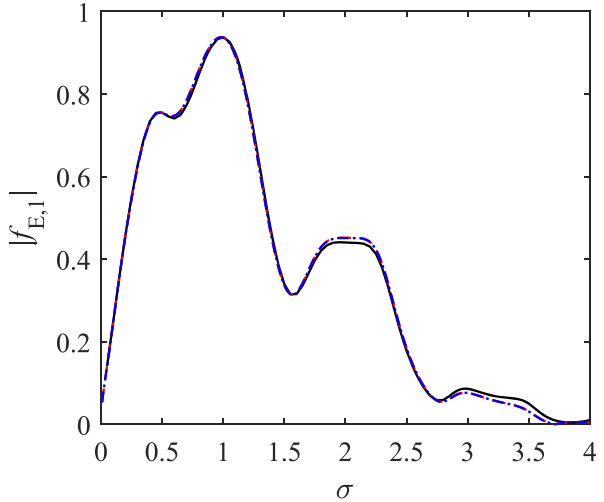

(a)

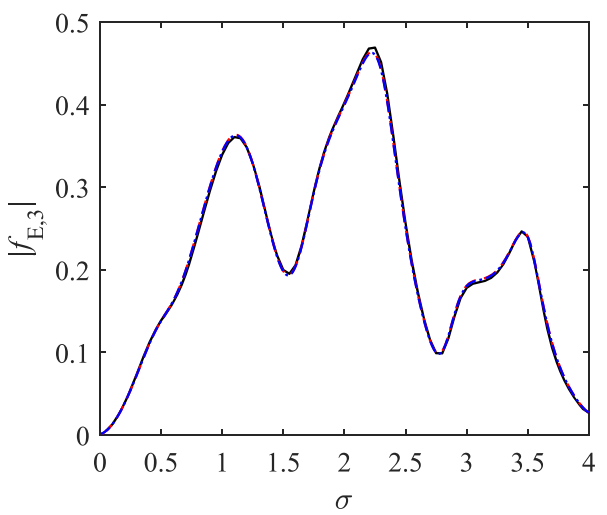

(c)

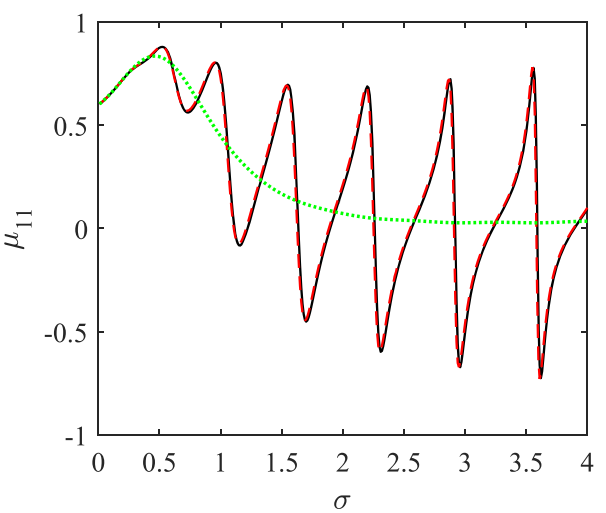

(a)

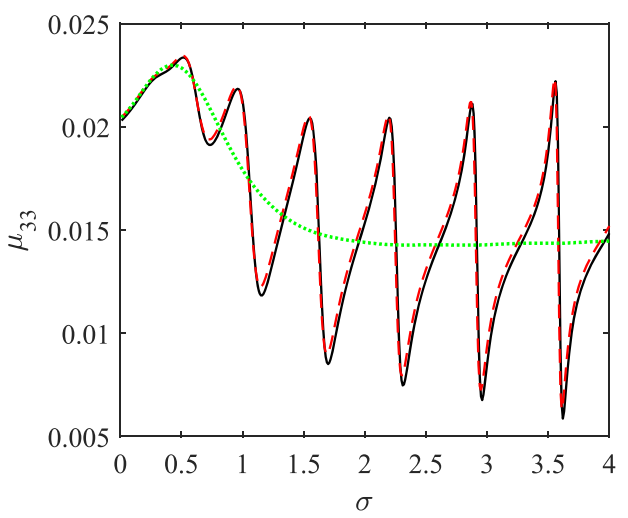

(c)

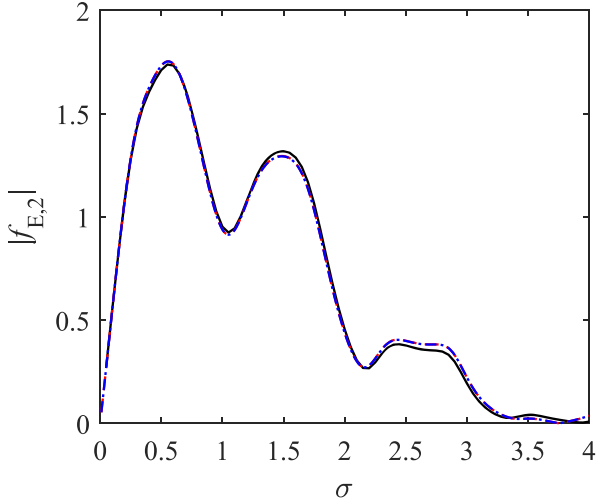

(b)
FIG. 7. Wave exciting force on an elliptic cylinder. (a) Sway; (b) heave; (c) roll. Solid lines: results computed by the hybrid method in the work of Li et al. $; 27$ dashed lines: results computed by the present method; dashed-dotted lines: same to dashed lines, but by the far field formula $\left(a=1, b=0.5,\left(x^{\prime}, z^{\prime}\right)=(0,-1)\right.$, $H=25, x_{1}=-x_{2}=-2.5, h_{1}=0.025$ and $h_{2}=0.1, d_{1}=0$ and $d_{2}=0, m_{1}=0.0225$ and $m_{2}=0.09$, and $L_{1}=0.0356$ and $\left.L_{2}=2.2791\right)$.

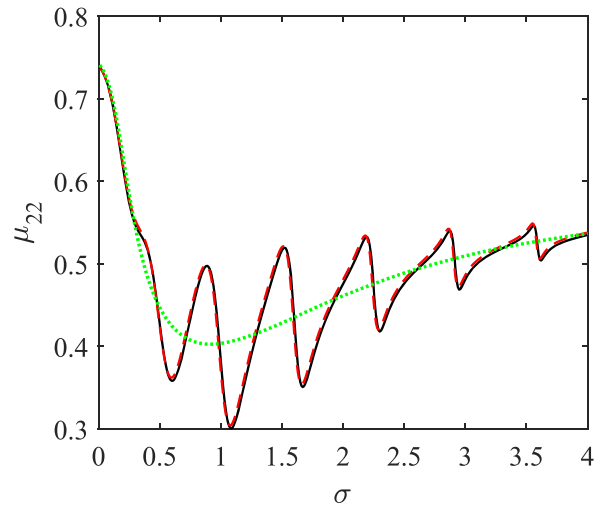

(b)

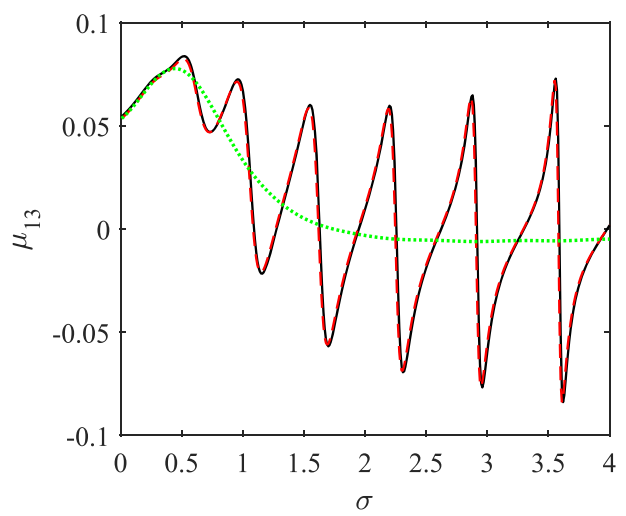

(d)
FIG. 8. Added mass of a floating rectangular body. (a) Sway; (b) heave; (c) roll; (d) sway-roll or roll-sway. Solid lines: semi-analytical solution in the work of Ren et al. ${ }^{25}$ dashed lines: results computed by the present method; dotted lines: results for open water $\left(a=1, b=0.5,\left(x^{\prime}, z^{\prime}\right)=(0,-b / 2)\right.$, $H=10, x_{1}=-x_{2}=-5, h_{1}=h_{2}=0.1$, $d_{1}=d_{2}=0.09, m_{1}=m_{2}=0.09$, and $L_{1}=L_{2}=4.5582$ ). 


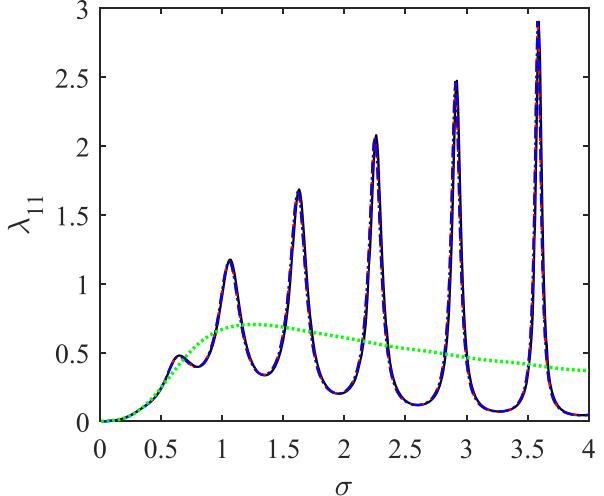

(a)



(c)

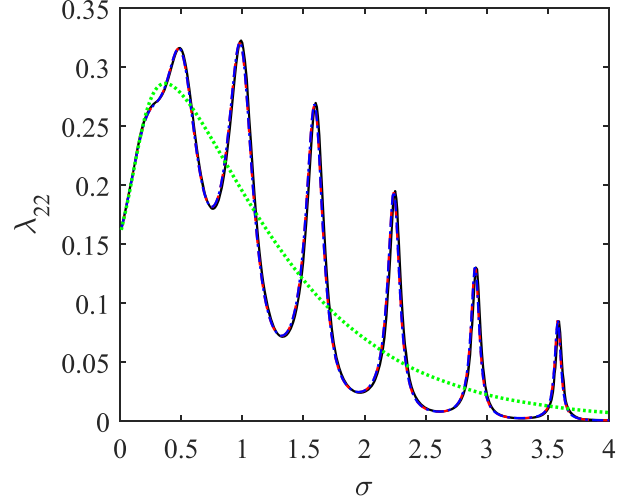

(b)

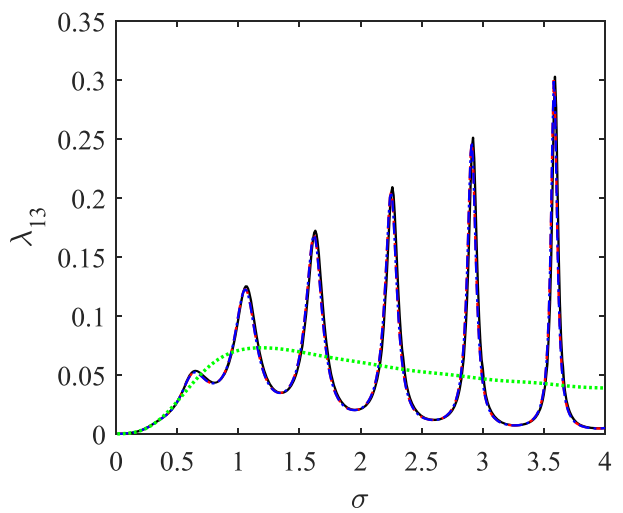

$(d)$

FIG. 9. Damping coefficient of a floating rectangular body. (a) Sway; (b) heave; (c) roll; (d) sway-roll or roll-sway. Solid lines: semi-analytical solution in the work of Ren et al. $;^{25}$ dashed lines: results computed by the present method; dashed-dotted lines: same to dashed lines, but by the far field formula; dotted lines: results for open water $\left(a=1, b=0.5,\left(x^{\prime}, z^{\prime}\right)=(0,-b / 2), H=10, x_{1}=-x_{2}=-5, h_{1}=h_{2}=0.1, d_{1}=d_{2}=0.09, m_{1}=m_{2}=0.09\right.$, and $\left.L_{1}=L_{2}=4.5582\right)$.

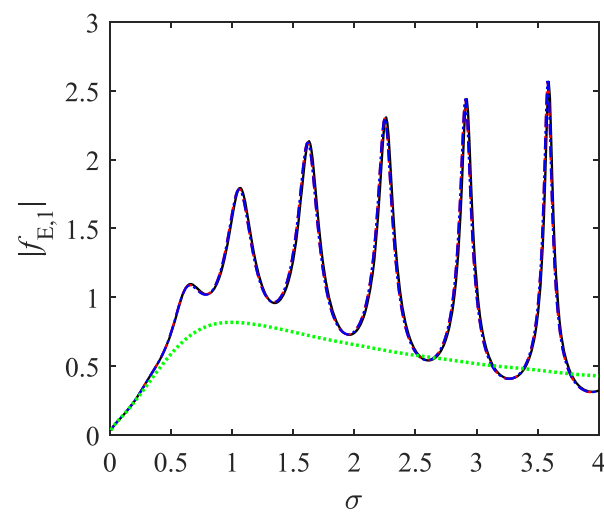

(a)

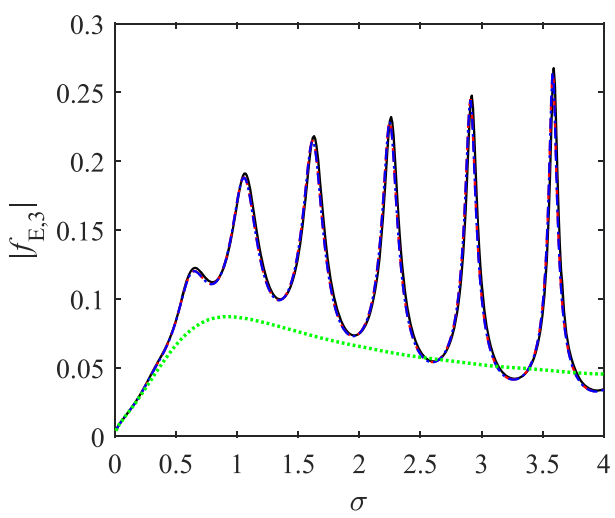

(c)

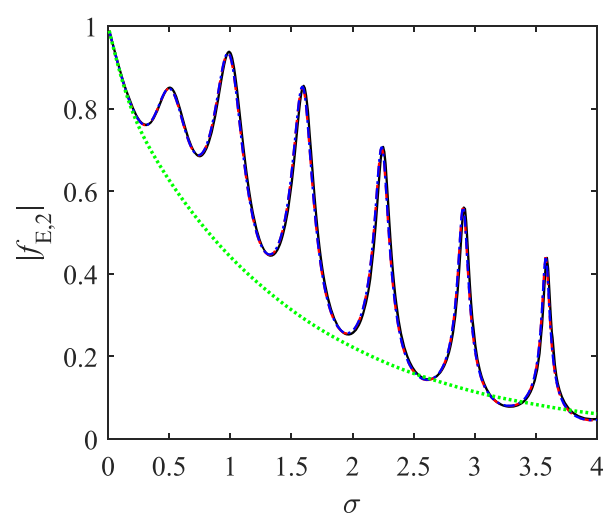

(b)
FIG. 10. Wave exciting force on a floating rectangular body. (a) Sway; (b) heave; (c) roll. Solid lines: semianalytical solution in the work of Ren et al. ${ }^{25}$ dashed lines: results computed by the present method; dashed-dotted lines: same to dashed lines, but by the far field formula; dotted lines: results for open water $\left(a=1, b=0.5,\left(x^{\prime}, z^{\prime}\right)=\right.$ $(0,-b / 2), H=10, x_{1}=-x_{2}=-5, h_{1}=$ $h_{2}=0.1, d_{1}=d_{2}=0.09, m_{1}=m_{2}=0.09$, and $L_{1}=L_{2}=4.5582$ ). 
us to use the explicit form of the derived formula to give some insights into the behaviours of the hydrodynamic force and body motion.

\section{Oscillation features of the hydrodynamic force}

Highly oscillatory behaviour of the hydrodynamic force has been observed in the wave/body/ice interaction problems, ${ }^{25}$ which is different from the typical case of a body floating on open water. From Eqs. (40) and (54), we can see that the oscillatory behaviour of the hydrodynamic force is closely linked to the coefficients $\varepsilon_{j}^{1}$ and $\varepsilon_{j}^{2}, \gamma_{1}$ and $\gamma_{2}$. If we look Eq. (30) carefully, we may see that the right-hand side terms related to $\varepsilon_{j}^{1}$ and $\varepsilon_{j}^{2}$ are due to the refection of the body generated wave by the ice sheet. The reflected wave will then be reflected back by the body to the ice sheet, which will be further reflected back to the body by the ice sheet. This resembles the sloshing wave inside a tank in which the waves continue to be reflected by the side walls, leading to the oscillatory behaviour.

We may use the case in Sec. III C as an example. Due to symmetry of the problem, we have $\varepsilon_{j}^{1}=(-1)^{j} \varepsilon_{j}^{2}$ and $f_{E, k}^{o+}=$ $(-1)^{k} f_{E, k}^{o-}$. The coefficients $\varepsilon_{j}^{2}$ in Eq. (36) can be written as

$$
\varepsilon_{j}^{2}=-\frac{\left(A_{j}^{+} t_{0}^{+}-A_{j}^{-} r_{0}^{+}\right) R_{L, 0}^{w 2 i} R_{R, 0}^{w 2 i} \mathrm{e}^{-k_{0} l}+A_{j}^{-} R_{L, 0}^{w 2 i}}{\left(t_{0}^{+} t_{0}^{-}-r_{0}^{+} r_{0}^{-}\right) R_{L, 0}^{w 2 i} R_{R, 0}^{w 2 i} \mathrm{e}^{-k_{0} l}-\mathrm{e}^{k_{0} l}+r_{0}^{-} R_{L, 0}^{w 2 i}+r_{0}^{+} R_{R, 0}^{w 2 i}},
$$

where $l=2 x_{2}=-2 x_{1}$. It is well known that with the increase of $\sigma$, we have $t_{0}^{+}=t_{0}^{-} \rightarrow 0$. Thus for relatively large $\sigma$, by

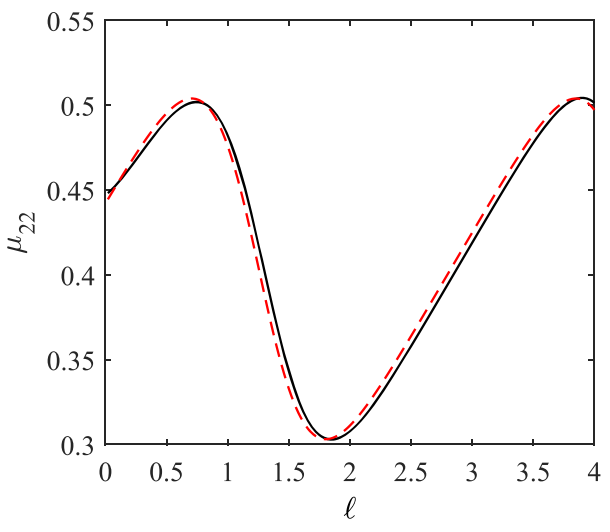

(a)

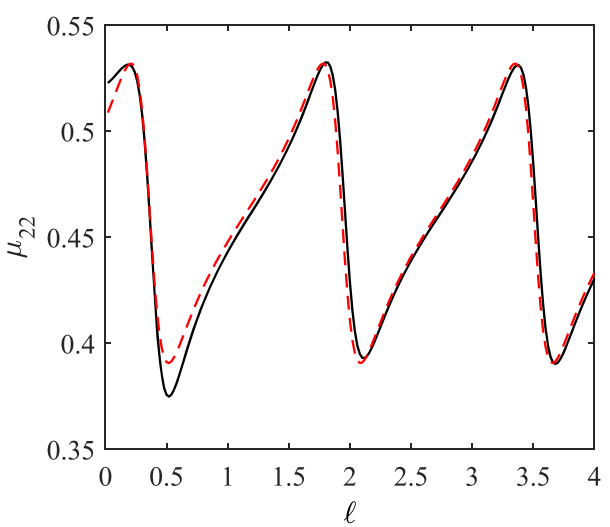

(c)

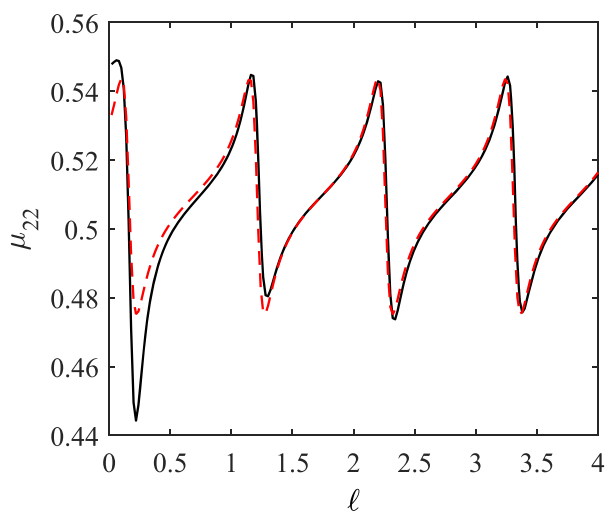

(e)

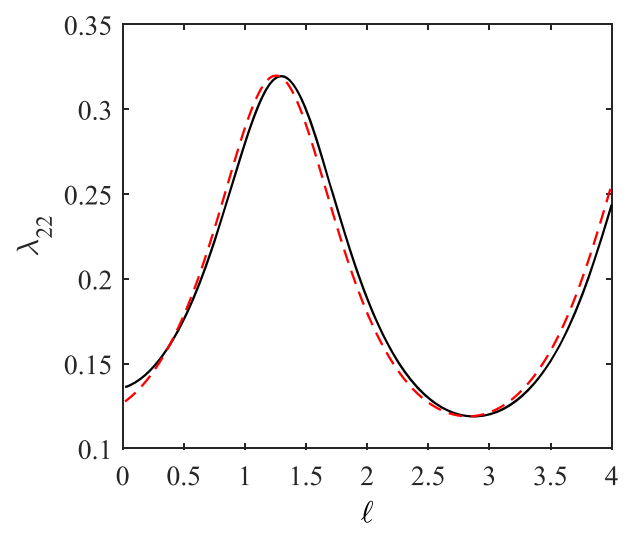

(b)

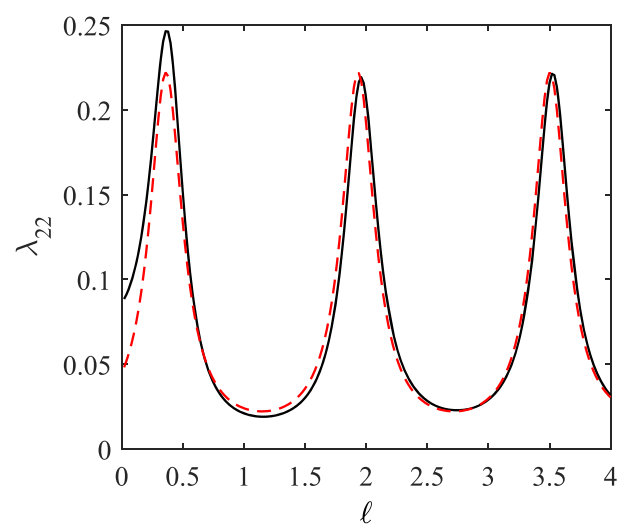

(d)

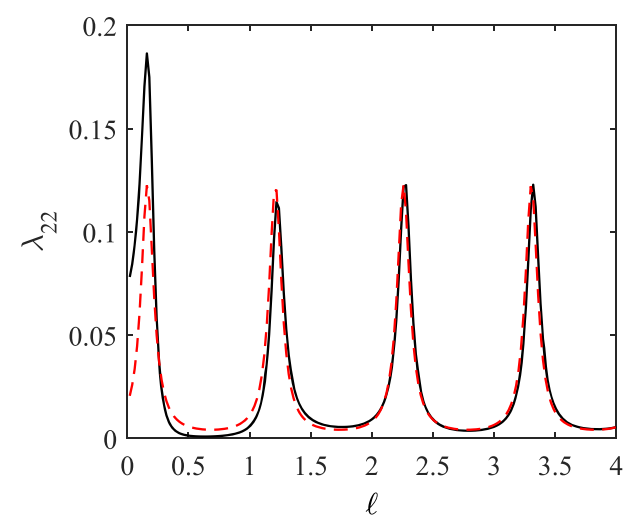

$(f)$
FIG. 11. Hydrodynamic coefficients of a floating rectangular body in the heave mode. In (a) and (b), $\sigma=1.0$; in (c) and (d), $\sigma=2.0$; in (e) and (f), $\sigma=$ 3.0. Solid lines: results computed by the exact method; dashed lines: results computed by the present method ( $a=1, b=$ $0.5,\left(x^{\prime}, z^{\prime}\right)=(0,-b / 2), H=10, h_{1}=h_{2}$ $=0.1, d_{1}=d_{2}=0.09, m_{1}=m_{2}=0.09$, and $L_{1}=L_{2}=4.5582$ ). 
letting $t_{0}^{+}=t_{0}^{-}=0$ Eq. (81) can be simplified as

$$
\varepsilon_{j}^{2}=\frac{A_{j}^{-} R}{\mathrm{e}^{k_{0} l}-r R},
$$

where $r=r_{0}^{+}=r_{0}^{-}$. Then invoking Eq. (82), we can find the peaks and troughs of $\left|\varepsilon_{j}^{2}\right|$ through

$$
\left|S_{\varepsilon}(\omega)\right|=\left|\mathrm{e}^{k_{0} l}-r R\right|=\sqrt{1+|r R|^{2}-2 \operatorname{Re}\left(r R \mathrm{e}^{-k_{0} l}\right)} .
$$

It shows that $\left|\varepsilon_{j}^{2}\right|$ will reach its peaks when $\delta=\mathrm{i} k_{0} l$ equals

$$
\delta_{P}^{\varepsilon}=2 n \pi-\beta-\operatorname{Arg}(r)
$$

and reach its troughs when $\delta$ equals

$$
\delta_{T}^{\mathcal{E}}=2 n \pi+\pi-\beta-\operatorname{Arg}(r),
$$

where $n$ includes all integers which ensure $\delta<0$ required based on the definition of $k_{0}$. From Eq. (58), the far field formula for $f_{E, k}^{\text {o- }}$ can be given as

$$
f_{E, k}^{0-}=-2 \mathrm{i} \rho \omega A_{k}^{-} C_{g},
$$

where $C_{g}$ is the wave group velocity in the open water. Substituting Eqs. (82) and (86) into Eq. (40), we have

$$
\mu_{k j}=\mu_{k j}^{\mathrm{o}}-\frac{2 \rho \omega C_{g}\left[1+(-1)^{j+k}\right]}{g} \operatorname{Im}\left(A_{k j}\right),
$$

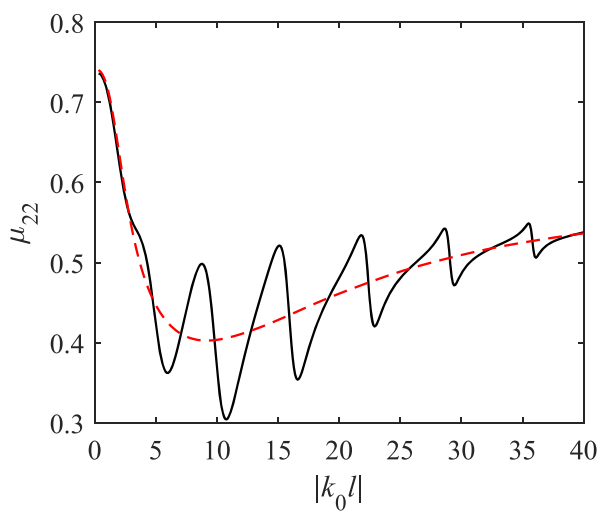

(a)

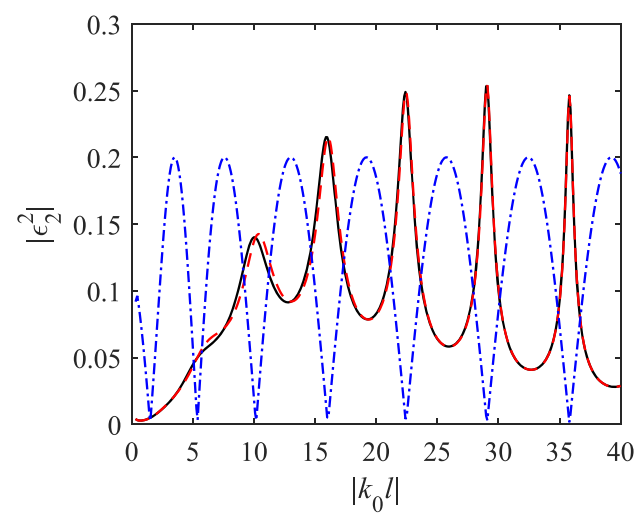

(c)

$$
\lambda_{k j}=\lambda_{k j}^{\mathrm{o}}-\frac{2 \rho \omega^{2} C_{g}\left[1+(-1)^{j+k}\right]}{g} \operatorname{Re}\left(A_{k j}\right)
$$

where

$$
\mathrm{A}_{k j}=\frac{A_{j}^{-} A_{k}^{-} R}{\mathrm{e}^{k_{0} l}-r R} .
$$

The denominator of this equation is the same as that of Eq. (82) and therefore Eqs. (84) and (85) apply here. From Eqs. (8.6.26) and (8.6.49) in the work of Mei et al., ${ }^{29}$ we have

$$
\operatorname{Arg}(r)=\operatorname{Arg}\left(t_{0}^{-}\right) \pm \pi / 2,
$$

and

$$
r+(-1)^{j} t_{0}^{-}=-\mathrm{e}^{2 \mathrm{i} \operatorname{Arg}\left(A_{j}^{-}\right)} .
$$

Thus when $\delta=\delta_{P}^{\varepsilon}$ in Eq. (84), we have

$$
\begin{aligned}
A_{k j} & =\frac{\left|A_{j}^{-} A_{k}^{-} R\right|}{1-|r R|} \mathrm{e}^{\mathrm{i}\left[\operatorname{Arg}\left(A_{j}^{-}\right)+\operatorname{Arg}\left(A_{k}^{-}\right)-\operatorname{Arg}(r)\right]} \\
& =\left\{\begin{array}{lc}
-\frac{\left|A_{j}^{-} A_{k}^{-} R\right|}{1-|r R|}\left[|r| \pm \mathrm{i}\left|t_{0}^{-}\right|\right], & j, k=1,3 \\
-\frac{\left|A_{j}^{-} A_{k}^{-} R\right|}{1-|r R|}\left[|r| \mp \mathrm{i}\left|t_{0}^{-}\right|\right], & j, k=2
\end{array},\right.
\end{aligned}
$$

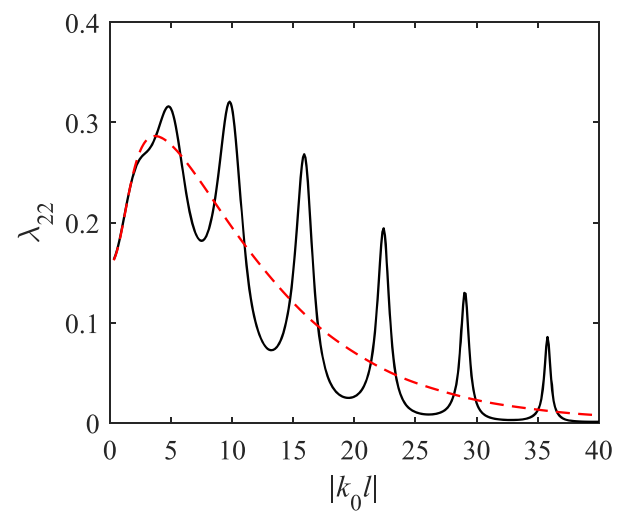

(b)

FIG. 12. Hydrodynamic coefficient in the heave mode against $\left|k_{0} l\right|$. (a) Added mass; (b) damping coefficient; (c) $\left|\varepsilon_{2}^{2}\right|$. In (a) and (b), solid lines are for polynya while dashed lines are for open water. In (c), the solid line is for Eq. (81), the dashed line is for Eq. (82), and the dasheddotted line represents $\left|S_{\varepsilon}(\omega)\right| / 10\left(a=1, b=0.5, H=10, x_{1}=-x_{2}=-5, h_{1}=h_{2}=0.1, d_{1}=d_{2}=0.09, m_{1}=m_{2}=0.09\right.$, and $L_{1}=L_{2}$ $=4.5582$ ). 
while when $\delta=\delta_{T}^{\varepsilon}$ in Eq. (85), we have

$$
\begin{aligned}
A_{k j} & =-\frac{\left|A_{j}^{-} A_{k}^{-} R\right|}{1+|r R|} \mathrm{e}^{\mathrm{i}\left[\operatorname{Arg}\left(A_{j}^{-}\right)+\operatorname{Arg}\left(A_{k}^{-}\right)-\operatorname{Arg}(r)\right]} \\
& =\left\{\begin{array}{ll}
\frac{\left|A_{j}^{-} A_{k}^{-} R\right|}{1+|r R|}\left[|r| \pm \mathrm{i}\left|t_{0}^{-}\right|\right], & j, k=1,3 \\
\frac{\left|A_{j}^{-} A_{k}^{-} R\right|}{1+|r R|}\left[|r| \mp \mathrm{i}\left|t_{0}^{-}\right|\right], & j, k=2
\end{array} .\right.
\end{aligned}
$$

It should be noticed that $t_{0}^{+}=t_{0}^{-} \rightarrow 0$ has been used for a large $\sigma$, in Eqs. (84) and (85), and it may be used in Eqs. (92) and (93) as well. Therefore, when $\lambda_{k j}$ will reach its peaks and troughs at $\delta=\delta_{P}^{\varepsilon}$ and $\delta=\delta_{T}^{\varepsilon}$, respectively, $\mu_{k j}$ will reach the value of $\mu_{k j}^{\mathrm{o}}$, or the last term in Eq. (87) due to which the ice sheet has no effect. These results can be seen in Fig. 12. We should also notice that Eqs. (87) and (88) are for a body of symmetry. $\mu_{k j}=0$ and $\lambda_{k j}=0$ when $k+j$ is an odd number, and therefore only even $k+j$ is discussed.

Invoking Eq. (40), we can see that the hydrodynamic coefficients will follow the oscillatory behaviour of $\varepsilon_{j}^{2}$ and the oscillation period in terms of $\left|k_{0} l\right|$ roughly equals $2 \pi$. This is reflected in Fig. 12, but the period of $2 \pi$ is not exact as other parameters in Eq. (81) vary with $\sigma$ or $k_{0}$ when $l$ is fixed. Thus in Fig. 13, results are plotted against $\left|k_{0} l\right|$ at various given $\sigma$ while varying $l$. From the figure, it can be seen that the period is $2 \pi$, as expected from Eq. (81).

For the wave exciting force, Eq. (54) can be rewritten as

$$
f_{E, k}=\Upsilon_{k} f_{E, k}^{\mathrm{o}-} \text {, }
$$

where

$$
\begin{aligned}
\Upsilon_{k} & =\gamma_{1}(-1)^{k}+\gamma_{2} \\
& =-\frac{T_{L, 0}^{i 2 w}\left[\left(t_{0}^{-}(-1)^{k}-r_{0}^{+}\right) R_{R, 0}^{w 2 i} \mathrm{e}^{-k_{0} l / 2}+\mathrm{e}^{k_{0} l / 2}\right]}{\left(t_{0}^{-} t_{0}^{+}-r_{0}^{-} r_{0}^{+}\right) R_{L, 0}^{w 2 i} R_{R, 0}^{w 2 i} \mathrm{e}^{-k_{0} l}-\mathrm{e}^{k_{0} l}+r_{0}^{-} R_{L, 0}^{w 2 i}+r_{0}^{+} R_{R, 0}^{w 2 i}} .
\end{aligned}
$$

Similar to Eq. (82), $\Upsilon_{k}$ can be approximated as

$$
\Upsilon_{k}=\frac{T_{L, 0}^{i 2 w}}{\mathrm{e}^{k_{0} l / 2}-r R \mathrm{e}^{-k_{0} l / 2}} .
$$

The peaks and troughs of $\left|\Upsilon_{k}\right|$ can be found through Eq. (83). Thus the oscillatory behaviour of the exciting force is the same as that of the hydrodynamic coefficient, as can be seen in Fig. 14 for the heave wave exciting force, together with Fig. 12 for the heave added mass and damping coefficient. Equation (95) indicates that $f_{E, k}$ will oscillate against $\left|k_{0} l\right|$ periodically at a given $\sigma$, as plotted in Fig. 15.

From Eqs. (81) and (95), it can be seen that the oscillatory behaviour for the hydrodynamic force will never diminish even when $l \rightarrow \infty$ at a given $\sigma$. This means that the motion of a body in an infinitely large polynya is not the same as that of a body on a completely open free surface. This may seem to be a surprise. However, we may notice the radiation conditions in these two cases are given in Eqs. (9), (10), (16), and (17), respectively, which are different. Different radiation conditions are expected to give different results. Physically, a periodic motion state can be reached only after $t \rightarrow \infty$. Thus no matter how large $l$ is, after sufficiently large time, the wave will arrive at the ice edges which will give wave reflection. The reflected wave will eventually affect the body. We may also notice that if we increase $\sigma$ at a fixed $l$ while $\exp \left(k_{0} l\right)$ is fixed, the result will be different. This is because $A_{j}^{-}, T_{L, 0}^{i 2 w}$, $R$, and $r$ will vary as well now. In particular, as $\sigma$ increases, for radiation problems, $A_{j}^{-}$will tend to zero and the effect of the ice sheet will disappear. For scattering problems, $T_{L, 0}^{i 2 w}$ will also tend to zero as $\sigma$ increases, which means that no wave will transmit into the polynya, and the exciting force will become zero.

From Fig. 10 in the work of Ren et al. ${ }^{25}$ it was found that there was no standing wave in the polynya due to the forced oscillation of the body. This can be analyzed explicitly through Eq. (30). On the left-hand side of the body, the complex wave amplitude of the wave along the $x$-axis is

$$
C_{k}=\varepsilon_{k}^{2}
$$

and that opposite to the $x$-axis is

$$
D_{k}=A_{k}^{-}+(-1)^{i} \varepsilon_{k}^{2} t_{0}^{+}+\varepsilon_{k}^{2} r_{0}^{-} .
$$

Invoking Eq. (82), when $\sigma$ increases, the above two equations can be further written as

$$
\begin{aligned}
C_{k} & =\frac{A_{k}^{-} R}{\mathrm{e}^{k_{0} l}-r R}, \\
D_{k} & =\frac{A_{k}^{-} \mathrm{e}^{k_{0} l}}{\mathrm{e}^{k_{0} l}-r R} .
\end{aligned}
$$

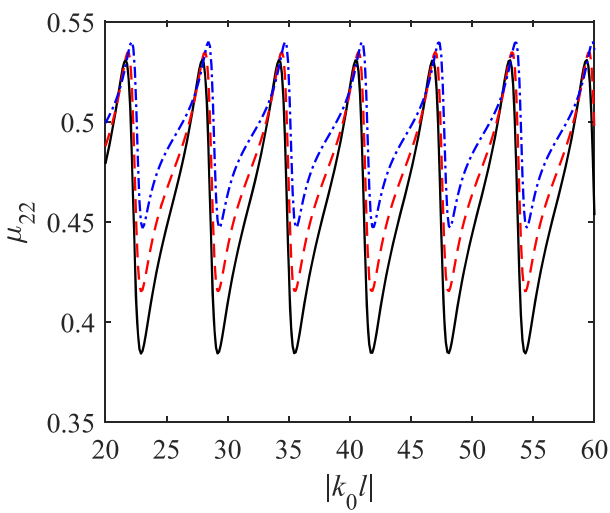

(a)

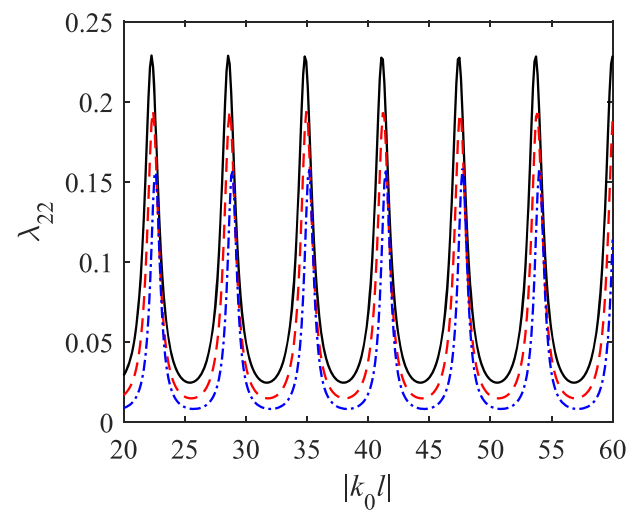

(b)
FIG. 13. Hydrodynamic coefficient in the heave mode with different $\sigma$ against $\left|k_{0} l\right|$. (a) Added mass; (b) damping coefficient. Solid lines: $\sigma=1.94$; dashed lines: $\sigma=2.24$; dashed-dotted lines: $\sigma=2.60(a=1, b=0.5, H=10$, $x_{1}=-x_{2}=-5, h_{1}=h_{2}=0.1, d_{1}=d_{2}$ $=0.09, m_{1}=m_{2}=0.09$, and $L_{1}=L_{2}=$ 4.5582). 


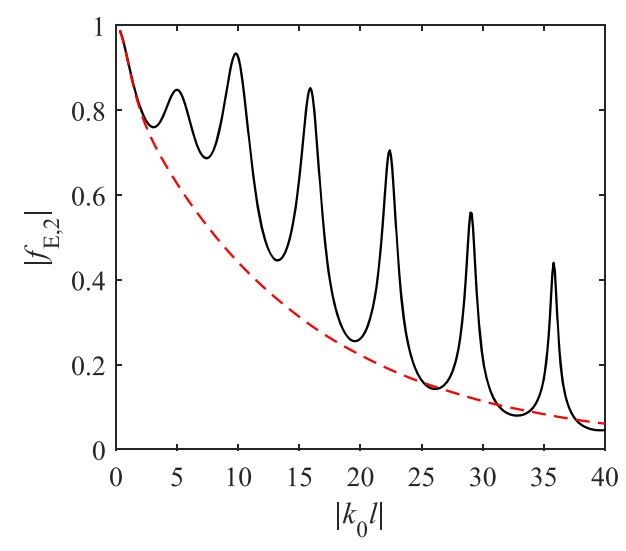

FIG. 14. Heave exciting force against $\left|k_{0} l\right|$. The solid line is for the polynya while the dashed line is for open water $\left(a=1, b=0.5, H=10, x_{1}=-x_{2}=-5\right.$, $h_{1}=h_{2}=0.1, d_{1}=d_{2}=0.09, m_{1}=m_{2}=0.09$, and $\left.L_{1}=L_{2}=4.5582\right)$.

This indicates that we generally have $\left|C_{k}\right|<\left|D_{k}\right|$ unless when $|R|=1$ at a total reflection which is most rare. Thus there is usually no standing wave in the polynya due to the forced motion.

For the scattering problem, Eq. (46) shows that on the left-hand side of the body, the complex wave amplitude for the waves along and opposite to the $x$-axis are

$$
\begin{gathered}
C_{0}=\gamma_{2}, \\
D_{0}=\gamma_{1} t_{0}^{+}+\gamma_{2} r_{0}^{-},
\end{gathered}
$$

respectively. Then invoking Eq. (96), the above two equations can be written as

$$
\begin{aligned}
& C_{0}=\frac{T_{L, 0}^{i 2 w}}{\mathrm{e}^{k_{0} l / 2}-r R \mathrm{e}^{-k_{0} l / 2}}, \\
& D_{0}=\frac{T_{L, 0}^{i 2 w} r_{0}^{-}}{\mathrm{e}^{k_{0} l / 2}-r R \mathrm{e}^{-k_{0} l / 2}} .
\end{aligned}
$$

At large $\sigma$, we have $|r|=\left|r_{0}^{+}\right|=\left|r_{0}^{-}\right| \rightarrow 1$, which gives $\left|C_{0}\right|=\left|D_{0}\right|$. Thus there could be standing waves or at least approximately.

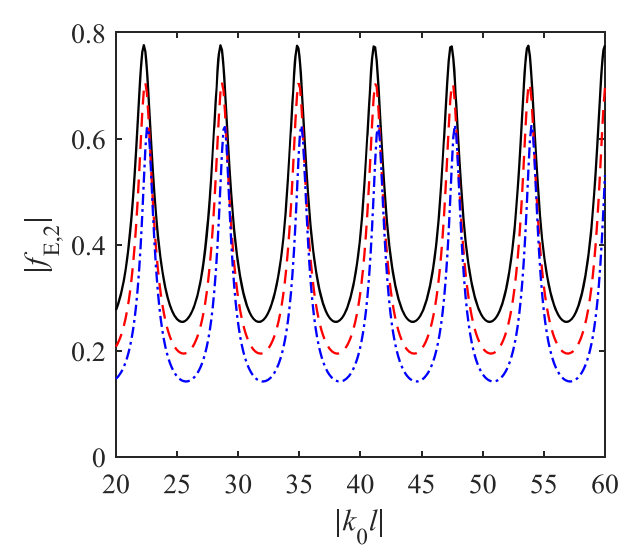

FIG. 15. Heave exciting force with different $\sigma$ against $\left|k_{0} l\right|$. Solid line: $\sigma=1.94$; dashed line: $\sigma=2.24$; dashed-dotted line: $\sigma=2.60(a=1, b=0.5$, $H=10, x_{1}=-x_{2}=-5, h_{1}=h_{2}=0.1, d_{1}=d_{2}=0.09, m_{1}=m_{2}=0.09$, and $L_{1}=L_{2}=4.5582$ ).

\section{Oscillation features of the body motion}

Since the body is symmetric about $x=0$, the symmetric heave motion is fully decoupled from the anti-symmetric sway and roll motions. From Eq. (14), the complex heave motion amplitude can be obtained as

$$
\frac{\alpha_{2}}{\alpha_{0}}=\frac{f_{E, 2}}{-\sigma\left(m_{22}+\mu_{22}\right)+\mathrm{i} \sqrt{\sigma} \lambda_{22}+C_{22}},
$$

where the parameters are all nondimensional. Invoking Eqs. (40) and (54), this becomes

$$
\frac{\alpha_{2}}{\alpha_{0}}=\frac{\left(\gamma_{1}+\gamma_{2}\right) f_{E, 2}^{\mathrm{o}-}}{-\sigma\left(m_{22}+\mu_{22}^{0}\right)+\mathrm{i} \sqrt{\sigma} \lambda_{22}^{0}+2 \sigma \varepsilon_{2}^{2} f_{E, 2}^{\mathrm{o}-}+C_{22}} .
$$

Resonance occurs when the exciting frequency coincides with one of the natural frequencies. For the undamped heave motion, we can find the natural frequencies when the inertial force is canceled by the restoring force or

$$
-\sigma\left[m_{22}+\mu_{22}^{0}-2 \operatorname{Re}\left(\varepsilon_{2}^{2} f_{E, 2}^{\mathrm{o}-}\right)\right]+C_{22}=0 .
$$

This equation shows that the natural frequency for heave motion in a polynya will be different from that for open water. Since both $\mu_{22}^{0}$ and $\varepsilon_{2}^{2} f_{E, 2}^{\mathrm{o}-}$ are frequency dependent, Eq. (107) has to be solved numerically, for example, done in Fig. 8(a) in the work of Ren et al. ${ }^{25}$ From the numerical solution, it is found that the natural frequency $\sigma \approx 1.19$ in the present case. A large peak can be found near this frequency (it should also be noticed that the damping will have some effect on this frequency) or resonance occurs. In addition to this peak, there are a series of local peaks in Fig. 16(a), which are not commonly seen in the open water, as reflected by the dashed line in the figure. For large $\sigma$, we may use Eqs. (82) and (96) in (106). This gives

$$
\frac{\alpha_{2}}{\alpha_{0}}=\frac{T_{L, 0}^{i 2 w} \tilde{\alpha}_{2} / \alpha_{0}}{\mathrm{e}^{k_{0} l / 2}-R\left(r-2 \sigma A_{2}^{-} \tilde{\alpha}_{2} / \alpha_{0}\right) \mathrm{e}^{-k_{0} l / 2}},
$$

where $\tilde{\alpha}_{2}$ is the complex heave motion for open water. Then the local extrema of $\left|\alpha_{2}\right| / \alpha_{0}$ can be found through the following equation:

$$
\begin{aligned}
& \left|U_{2}(\sigma)\right|=\left|\mathrm{e}^{k_{0} l / 2}-R\left(r-2 \sigma A_{2}^{-} \tilde{\alpha}_{2} / \alpha_{0}\right) \mathrm{e}^{-k_{0} l / 2}\right| \\
& =\sqrt{1+\left|R\left(r-2 \sigma A_{2}^{-} \tilde{\alpha}_{2} / \alpha_{0}\right)\right|^{2}-2 \operatorname{Re}\left[R\left(r-2 \sigma A_{2}^{-} \tilde{\alpha}_{2} / \alpha_{0}\right) \mathrm{e}^{-k_{0} l}\right]},
\end{aligned}
$$

or more directly through

$$
\left|S_{2}(\sigma)\right|=\left|1+\mathrm{e}^{-k_{0} l} \mathrm{e}^{\mathrm{i} \operatorname{Arg}\left[R\left(r-2 \sigma A_{2}^{-} \tilde{\alpha}_{2} / \alpha_{0}\right)\right]}\right| .
$$

Its peaks and troughs occur when $\delta$ equals

$$
\delta_{P}^{2}=2 n \pi-\beta-\operatorname{Arg}\left(r-2 \sigma A_{2}^{-} \tilde{\alpha}_{2} / \alpha_{0}\right),
$$

and

$$
\delta_{T}^{2}=2 n \pi+\pi-\beta-\operatorname{Arg}\left(r-2 \sigma A_{2}^{-} \tilde{\alpha}_{2} / \alpha_{0}\right),
$$

respectively. $\left|S_{2}(\sigma)\right|$ is plotted in Fig. 16(a). It can be seen that oscillations of $\left|\alpha_{2}\right| / \alpha_{0}$ and $\left|S_{2}(\sigma)\right|$ follow the same peaks and troughs, which explains its oscillatory behaviour. Equation (108) also indicates that $\left|\alpha_{2}\right| / \alpha_{0}$ will oscillate with $\left|k_{0} l\right|$ periodically at a given $\sigma$ with 


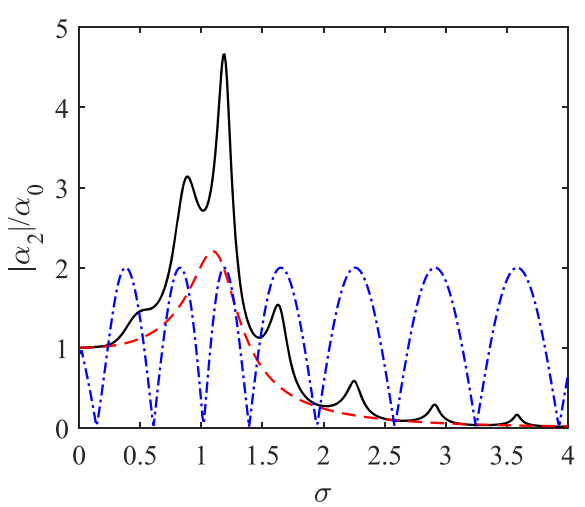

(a)

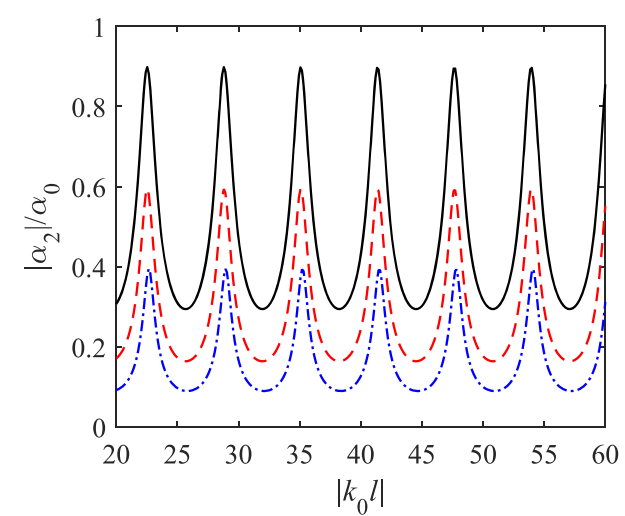

(b)

FIG. 16. Heave motion of a floating rectangle body. (a) $\left|\alpha_{2}\right| / \alpha_{0}$ against $\sigma$. (b) $\left|\alpha_{2}\right| / \alpha_{0}$ against $\left|k_{0} l\right|$. In (a), the solid line is for $x_{1}=-x_{2}=-5$ while the dashed line is for open water, the dashed-dotted line represents $\left|S_{2}(\sigma)\right|$. In (b), the solid line is for $\sigma=1.94$, the dashed line is for $\sigma=2.24$, the dasheddotted line is for $\sigma=2.60(a=1, b=$ $0.5, m_{22}=0.5, C_{22}=1, H=10, h_{1}=h_{2}$ $=0.1, d_{1}=d_{2}=0.09, m_{1}=m_{2}=0.09$, and $L_{1}=L_{2}=4.5582$ ).

the period of $2 \pi$, and the peaks and troughs occur when $\delta$ equals $\delta_{P}^{2}$ and $\delta_{T}^{2}$, respectively, as shown in Fig. 16(b).

For the anti-symmetric coupled sway and roll motions, Eq. (14) provides

$$
\begin{aligned}
& \frac{\alpha_{1}}{\alpha_{0}}=\frac{\vartheta_{1}}{\vartheta}, \\
& \frac{\alpha_{3}}{\alpha_{0}}=\frac{\vartheta_{3}}{\vartheta}
\end{aligned}
$$

with

$$
\begin{aligned}
\vartheta_{1}= & {\left[-\sigma m_{33}+\left(-\sigma \mu_{33}+\mathrm{i} \sqrt{\sigma} \lambda_{33}\right)+C_{33}\right] f_{E, 1} } \\
& -\left(-\sigma \mu_{13}+\mathrm{i} \sqrt{\sigma} \lambda_{13}\right) f_{E, 3},
\end{aligned}
$$

$$
\begin{aligned}
\vartheta_{3}= & {\left[-\sigma m_{11}+\left(-\sigma \mu_{11}+\mathrm{i} \sqrt{\sigma} \lambda_{11}\right)\right] f_{E, 3} } \\
& -\left(-\sigma \mu_{31}+\mathrm{i} \sqrt{\sigma} \lambda_{31}\right) f_{E, 1},
\end{aligned}
$$

$$
\begin{aligned}
\vartheta= & {\left[-\sigma m_{11}+\left(-\sigma \mu_{11}+\mathrm{i} \sqrt{\sigma} \lambda_{11}\right)\right]\left[-\sigma m_{33}\right.} \\
& \left.+\left(-\sigma \mu_{33}+\mathrm{i} \sqrt{\sigma} \lambda_{33}\right)+C_{33}\right] \\
& -\left(-\sigma \mu_{13}+\mathrm{i} \sqrt{\sigma} \lambda_{13}\right)\left(-\sigma \mu_{31}+\mathrm{i} \sqrt{\sigma} \lambda_{31}\right) .
\end{aligned}
$$

Then invoking Eq. (40) the undamped natural frequency can be found through

$$
\begin{aligned}
\sigma\{[ & \left.-\left(m_{11}+\mu_{11}^{0}\right)+2 \operatorname{Re}\left(\varepsilon_{1}^{2} f_{E, 1}^{\mathrm{o}-}\right)\right]\left[-\left(m_{33}+\mu_{33}^{0}\right)+2 \operatorname{Re}\left(\varepsilon_{3}^{2} f_{E, 3}^{\mathrm{o}-}\right)\right] \\
& \left.-\left[-\mu_{13}^{0}+2 \operatorname{Re}\left(\varepsilon_{3}^{2} f_{E, 1}^{\mathrm{o}-}\right)\right]\left[-\mu_{31}^{0}+2 \operatorname{Re}\left(\varepsilon_{1}^{2} f_{E, 3}^{\mathrm{o}-}\right)\right]\right\} \\
& +C_{33}\left[-\left(m_{11}+\mu_{11}^{0}\right)+2 \operatorname{Re}\left(\varepsilon_{1}^{2} f_{E, 1}^{\mathrm{o}-}\right)\right]=0
\end{aligned}
$$

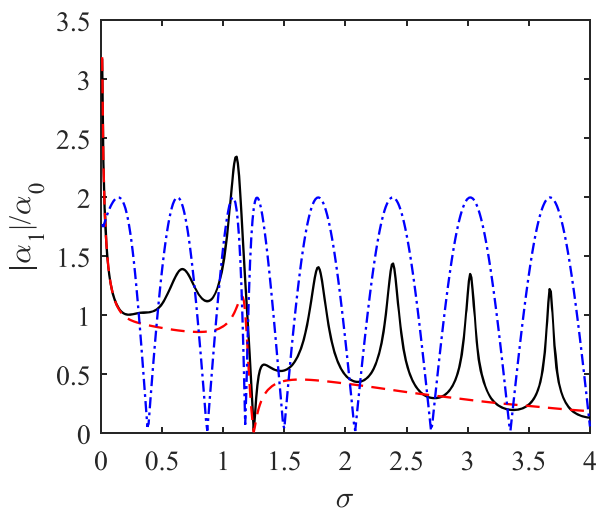

(a)

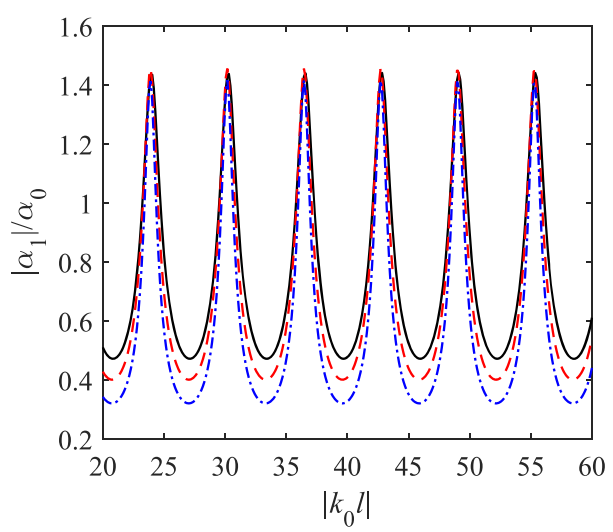

(c)

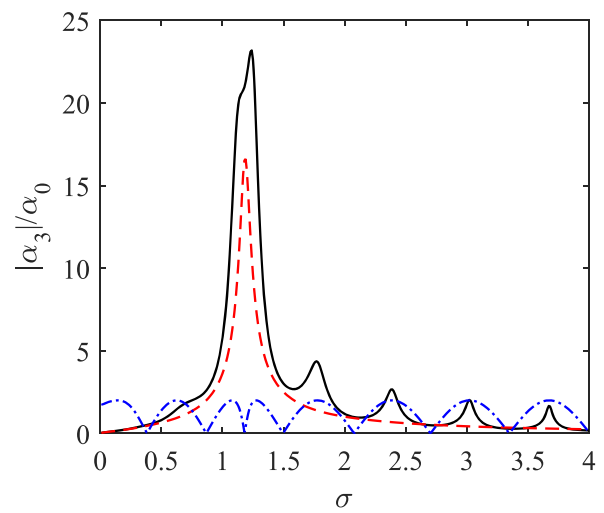

(b)

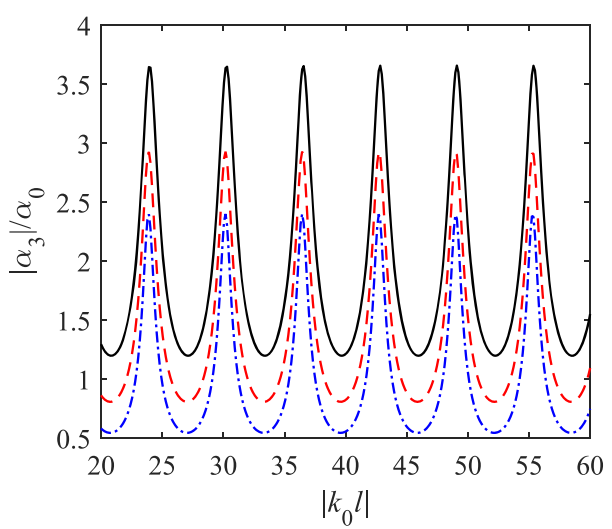

(d)
FIG. 17. Coupled sway and roll motions of a floating rectangle body. (a) $\left|\alpha_{1}\right| / \alpha_{0}$ against $\sigma$. (b) $\left|\alpha_{3}\right| / \alpha_{0}$ against $\sigma$. (c) $\left|\alpha_{1}\right| / \alpha_{0}$ against $\left|k_{0} l\right|$. (d) $\left|\alpha_{3}\right| / \alpha_{0}$ against $\left|k_{0} l\right|$. In (a) and (b), the solid line is for $x_{1}=-x_{2}=-5$ while the dashed line is for open water, the dashed-dotted line represents $\left|S_{13}(\sigma)\right|$. In (c) and (d), the solid line is for $\sigma=$ 1.94, the dashed line is for $\sigma=2.24$, the dashed-dotted line is for $\sigma=2.60$ $\left(a=1, b=0.5, m_{11}=0.5, m_{33}=0.0521\right.$, $C_{33}=1 / 12, H=10, h_{1}=h_{2}=0.1, d_{1}=$ $d_{2}=0.09, m_{1}=m_{2}=0.09$, and $L_{1}=L_{2}$ $=4.5582$ ). 
The numerical solution of this equation shows that there are multi-natural frequencies for the coupled motions. Especially near $\sigma=1.26$, the coupled motions are very large due to which the equivalent damping level is very small at this frequency. Similar to the heave motion, there are also a series of local peaks and troughs in $\left|\alpha_{1}\right| / \alpha_{0}$ and $\left|\alpha_{3}\right| / \alpha_{0}$, which can be analyzed by substituting Eqs. (82) and (96) into Eqs. (113) and (114) or

$$
\begin{aligned}
& \frac{\alpha_{1}}{\alpha_{0}}=\frac{T_{L, 0}^{i 2 w} \tilde{\alpha}_{1} / \alpha_{0}}{\mathrm{e}^{k_{0} l / 2}-\left[R r-2 R \sigma\left(A_{1}^{-} \tilde{\alpha}_{1} / \alpha_{0}+A_{3}^{-} \tilde{\alpha}_{3} / \alpha_{0}\right)\right] \mathrm{e}^{-k_{0} l / 2}}, \\
& \frac{\alpha_{3}}{\alpha_{0}}=\frac{T_{L, 0}^{i 2 w} \tilde{\alpha}_{3} / \alpha_{0}}{\mathrm{e}^{k_{0} l / 2}-\left[R r-2 R \sigma\left(A_{1}^{-} \tilde{\alpha}_{1} / \alpha_{0}+A_{3}^{-} \tilde{\alpha}_{3} / \alpha_{0}\right)\right] \mathrm{e}^{-k_{0} l / 2}} .
\end{aligned}
$$

Similar to Eq. (108), the local extrema for the coupled motions can be found through

$\left|S_{13}(\sigma)\right|=\left|1+\mathrm{e}^{-k_{0} l} \mathrm{e}^{\mathrm{i} \operatorname{Arg}\left[R r-2 R \sigma\left(A_{1}^{-} \tilde{\alpha}_{1} / \alpha_{0}+A_{3}^{-} \tilde{\alpha}_{3} / \alpha_{0}\right)\right]}\right|$.

Its peaks and troughs occur when $\delta$ equals

$\delta_{P}^{13}=2 n \pi-\beta-\operatorname{Arg}\left[r-2 \sigma\left(A_{1}^{-} \tilde{\alpha}_{1} / \alpha_{0}+A_{3}^{-} \tilde{\alpha}_{3} / \alpha_{0}\right)\right]$,

and

$$
\delta_{T}^{13}=2 n \pi+\pi-\beta-\operatorname{Arg}\left[r-2 \sigma\left(A_{1}^{-} \tilde{\alpha}_{1} / \alpha_{0}+A_{3}^{-} \tilde{\alpha}_{3} / \alpha_{0}\right)\right],
$$

respectively. It can be seen from Figs. 17(a) and 17(b) that $\left|\alpha_{1}\right| / \alpha_{0}$ and $\left|\alpha_{3}\right| / \alpha_{0}$ follow the same peaks and troughs as $\left|S_{13}(\sigma)\right|$. Equations (119) and (120) indicate that $\left|\alpha_{1}\right| / \alpha_{0}$ and $\left|\alpha_{3}\right| / \alpha_{0}$ will also oscillate against $\left|k_{0} l\right|$ for a given $\sigma$ with period as $2 \pi$, and the peaks and troughs appear when $\delta$ equals $\delta_{P}^{13}$ and $\delta_{T}^{13}$, respectively, as reflected in Figs. 17(c) and 17(d).

\section{CONCLUSIONS}

A method based on the wide spacing approximation has been proposed for the interaction of water wave with a body floating on a polynya. It has been found that this method based on the solutions for a floating body without an ice sheet and for an ice sheet/free surface without a floating body can give accurate results for wave/body/ice sheet interaction problems. Extensive numerical results are provided, including the wave propagation across the polynya and wave interaction with a submerged body and a floating body in polynya. The complex wave features, as well as the hydrodynamic force and body response to the waves, are analyzed. From these the following conclusions can be drawn:

(1) The method is accurate and efficient for the problems of wave propagation across a polynya, and interaction with the submerged and floating bodies in polynya, including the long wave cases even though the method is based on the assumption of short waves.

(2) An explicit formula based on the present approximation has been found, which provides the discrete frequencies at which the wave reflection from a polynya confined between two semi-infinite ice sheets is zero. It occurs when the wavenumber $K$ nondimensionalized by polynya width $l$ is at $K l=n \pi+\operatorname{Arg}(R)$, where $n$ includes all integers which ensure $K l>0$ and $R$ is the complex reflection coefficient of the free surface water wave by the semi-infinite ice sheet.

(3) The hydrodynamic force on a body in a polynya has a highly oscillatory behaviour with the variation of the frequency. The mechanism for such oscillation has been investigated, which is found to be principally due to the waves being constantly reflected between the body and the ice sheet. It has been found that when $K l=2 n \pi+\operatorname{Arg}(R r)$ or $K l=2 n \pi+\pi+\operatorname{Arg}(R r)$, where $r$ is the reflection coefficient of the body in the open water without ice, the damping coefficient and wave exciting force tend to peak and trough values, respectively, while the added mass tends to the value in the open water.

(4) The body motion in a polynya, excited by an incoming wave, can experience resonance as in the open water, although the resonant frequency is different. In addition to the peak at the resonance, the motion amplitude also has many local peaks, or it also has oscillatory behaviour with respect to the frequency. These peaks are not necessarily due to the resonance at which the inertial force is canceled by the restoring force. They are primarily linked to the oscillatory behaviours of the hydrodynamic coefficients and the excitation force, although their peaks and troughs may not be at the same frequency.

(5) At a given frequency, the hydrodynamic force and motion response of a body in polynya will vary with the polynya width periodically, and the period is $\Delta l=2 \pi / K$. This suggests that no matter how wide the polynya is, the effect of the ice sheet always exists. It means that when $l \rightarrow \infty$, the result does not tend to that in the open water without ice.

\section{ACKNOWLEDGMENTS}

This work is supported by Lloyd's Register Foundation through the joint centre involving University College London, Shanghai Jiaotong University, and Harbin Engineering University, to which the authors are most grateful. Lloyd's Register Foundation helps to protect life and property by supporting engineering-related education, public engagement, and the application of research. This work is also supported by the National Natural Science Foundation of China (Grant No. 11472088).

\footnotetext{
${ }^{1}$ V. A. Squire, "Past, present and impendent hydroelastic challenges in the polar and subpolar seas," Philos. Trans. 369, 2813 (2011).

${ }^{2}$ E. M. Appolonov, K. E. Sazonov, A. A. Dobrodeev, N. Y. Klementieva, M. A. Kudrin, E. A. Maslich, V. O. Petinov, and V. M. Shaposhnikov, "Studies for development of technologies to make a wide channel in ice," in The 22nd International Conference on Port and Ocean Engineering under Arctic Conditions, Espoo, Finland, 9-13 June 2013.

${ }^{3}$ G. D. Q. Robin, "Wave propagation through fields of pack ice," Philos. Trans. R. Soc., A 255, 313 (1963).
} 
${ }^{4}$ V. A. Squire, J. P. Dugan, P. Wadhams, P. J. Rottier, and A. K. Liu, "Of ocean waves and sea ice," Annu. Rev. Fluid Mech. 27, 115 (1995).

${ }^{5}$ V. A. Squire, "Of ocean waves and sea-ice revisited," Cold Reg. Sci. Technol. 49, 110 (2007).

${ }^{6}$ C. Fox and V. A. Squire, "Reflection and transmission characteristics at the edge of shore fast sea ice," J. Geophys. Res.: Oceans 95, 11629, doi:10.1029/jc095ic07p11629 (1990).

${ }^{7}$ C. Fox and V. A. Squire, "Coupling between the ocean and an ice shelf," Ann. Glaciol. 15, 101 (1991).

${ }^{8}$ C. Fox and V. A. Squire, "On the oblique reflexion and transmission of ocean waves at shore fast sea ice," Philos. Trans. R. Soc., A 347, 185 (1994).

${ }^{9}$ T. Sahoo, T. L. Yip, and A. T. Chwang, "Scattering of surface waves by a semi-infinite floating elastic plate," Phys. Fluids 13, 3215 (2001).

${ }^{10}$ M. Meylan and V. A. Squire, "The response of ice floes to ocean waves," J. Geophys. Res.: Atmos. 99, 891, doi:10.1029/93jc02695 (1994).

${ }^{11}$ D. V. Evans and T. V. Davies, "Wave-ice interaction," Report No. 1313, Davidson Laboratory, Stevens Institute of Technology, New Jersey, 1968.

${ }^{12}$ H. Chung and C. Fox, "Calculation of wave-ice interaction using the WienerHopf technique," New Zealand J. Math. 31, 1 (2002).

${ }^{13}$ N. J. Balmforth and R. V. Craster, "Ocean waves and ice sheets," J. Fluid Mech. 395, 89 (1999).

${ }^{14}$ L. A. Tkacheva, "Hydroelastic behavior of a floating plate in waves," J. Appl. Mech. Tech. Phys. 42, 991 (2001).

${ }^{15} \mathrm{~L}$. A. Tkacheva, "The diffraction of surface waves by a floating elastic plate at oblique incidence," J. Appl. Math. Mech. 68, 425 (2004).

${ }^{16} \mathrm{H}$. Chung and C. M. Linton, "Reflection and transmission of waves across a gap between two semi-infinite elastic plates on water," Q. J. Mech. Appl. Math. 58, 1 (2005).

${ }^{17}$ T. D. Williams and V. A. Squire, "Scattering of flexural-gravity waves at the boundaries between three floating sheets with applications," J. Fluid Mech. 569, 113 (2006).

${ }^{18}$ D. V. Evans and R. Porter, "Wave scattering by narrow cracks in ice sheets floating on water of finite depth," J. Fluid Mech. 484, 143 (2003).

${ }^{19}$ R. Porter and D. Evans, "Diffraction of flexural waves by finite straight cracks in an elastic sheet over water," J. Fluids Struct. 23, 309 (2007).
${ }^{20}$ I. V. Sturova and L. A. Tkacheva, "Action of periodic external pressure on inhomogeneous ice cover," in The 32th International Workshop on Water Waves and Floating Bodies, Dalian, China, 23-26 April 2017.

${ }^{21}$ D. Das and B. N. Mandal, "Oblique wave scattering by a circular cylinder submerged beneath an ice-cover," Int. J. Eng. Sci. 44, 166 (2006).

${ }^{22}$ I. V. Sturova, "Wave generation by an oscillating submerged cylinder in the presence of a floating semi-infinite elastic plate," Fluid Dyn. 49, 504 (2014).

${ }^{23} \mathrm{I}$. V. Sturova, "The effect of a crack in an ice sheet on the hydrodynamic characteristics of a submerged oscillating cylinder," J. Appl. Math. Mech. 79, 170 (2015).

${ }^{24}$ I. V. Sturova, "Radiation of waves by a cylinder submerged in water with ice floe or polynya," J. Fluid Mech. 784, 373 (2015).

${ }^{25}$ K. Ren, G. X. Wu, and G. A. Thomas, "Wave excited motion of a body floating on water confined between two semi-infinite ice sheets," Phys. Fluids 28, 127101 (2016).

${ }^{26}$ Z. F. Li, Y. Y. Shi, and G. X. Wu, "Large amplitude motions of a submerged circular cylinder in water with an ice cover," Eur. J. Mech.-B/Fluids 65, 141 (2017).

${ }^{27}$ Z. F. Li, Y. Y. Shi, and G. X. Wu, "A hybrid method for wave interacting with a body floating on polynya confined between two semi-infinite ice sheets," in The 32th International Workshop on Water Waves and Floating Bodies, Dalian, China, 23-26 April 2017.

${ }^{28}$ M. A. Srokosz and D. V. Evans, "A theory for wave-power absorption by two independently oscillating bodies," J. Fluid Mech. 90, 337 (1979).

${ }^{29}$ C. C. Mei, M. Stiassnie, and K. P. Yue, Theory and Applications of Ocean Surface Waves Part 1: Linear Aspects (World Scientific Publishing Co. Pte. Ltd., Hackensack, NJ, 2005).

${ }^{30} \mathrm{R}$. W. Yeung, "A hybrid integral-equation method for time-harmonic freesurface flows," in The 1st International Conference on Numerical Ship Hydrodynamics, Gaithersburg, Maryland, 20-22 October 1975.

${ }^{31}$ R. Eatock Taylor and J. Zietsman, "A comparison of localized finite element formulations for two-dimensional wave diffraction and radiation problems," Int. J. Numer. Methods Eng. 17, 1355 (1981).

${ }^{32}$ Z. F. Li, Y. Y. Shi, and G. X. Wu, "Interaction of waves with a body floating on polynya between two semi-infinite ice sheets," J. Fluids Struct. (submitted).

${ }^{33}$ M. Meylan and V. A. Squire, "Finite-floe wave reflection and transmission coefficients from a semi-infinite model," J. Geophys. Res.: Atmos. 981, 12537, doi:10.1029/93jc00940 (1993). 